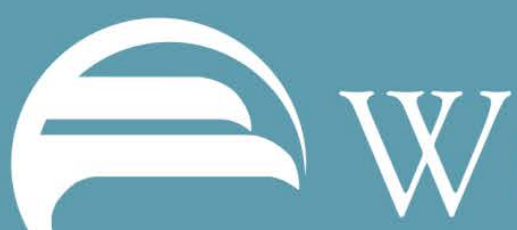

ORKING PAPERS

RESEARCH DEPARTMENT

\section{WORKING PAPER NO. 13-16 \\ IMPROVING GDP MEASUREMENT: A MEASUREMENT-ERROR PERSPECTIVE}

S. Borağan Aruoba

University of Maryland and Visiting Scholar, Federal Reserve Bank of Philadelphia

Francis X. Diebold

University of Pennsylvania and

Visiting Scholar, Federal Reserve Bank of Philadelphia

Jeremy Nalewaik

Federal Reserve Board of Governors

Frank Schorfheide

University of Pennsylvania and

Visiting Scholar, Federal Reserve Bank of Philadelphia

Dongho Song

University of Pennsylvania

First Draft: January 2013; This Draft: May 2, 2013

\section{Research Department, Federal Reserve Bank of Philadelphia}

Ten Independence Mall, Philadelphia, PA 19106-1574 • www.philadelphiafed.org/research-and-data/ 


\title{
Improving GDP Measurement: \\ A Measurement-Error Perspective
}

\author{
Borağan Aruoba* \\ University of Maryland \\ Jeremy Nalewaik \\ Federal Reserve Board \\ Francis X. Diebold \\ University of Pennsylvania \\ Frank Schorfheide \\ University of Pennsylvania \\ Dongho Song \\ University of Pennsylvania
}

First Draft, January 2013

This Draft, May 2, 2013

\begin{abstract}
We provide a new and superior measure of U.S. GDP, obtained by applying optimal signal-extraction techniques to the (noisy) expenditure-side and income-side estimates. Its properties - particularly as regards serial correlation - differ markedly from those of the standard expenditure-side measure and lead to substantially-revised views regarding the properties of GDP.
\end{abstract}

Key words: Income, Output, expenditure, business cycle, expansion, contraction, recession, turning point, state-space model, dynamic factor model, forecast combination JEL codes: E01, E32

Acknowledgments: For helpful comments we thank Bob Chirinko, Don Harding, Greg Mankiw, Adrian Pagan, John Roberts, Matt Shapiro, Chris Sims, Mark Watson, Justin Wolfers and Simon van Norden. For research support we thank the National Science Foundation and the Real-Time Data Research Center at the Federal Reserve Bank of Philadelphia. The views expressed in this paper are those of the authors and do not necessarily reflect the views of the Federal Reserve Bank of Philadelphia or the Federal Reserve System. This paper is available free of charge at www.philadelphiafed.org/research-and-data/publications/working-papers/.

\footnotetext{
${ }^{*}$ Contact Author: aruoba@econ.umd.edu
} 


\section{Introduction}

Aggregate real output is surely the most fundamental and important concept in macroeconomic theory. Surprisingly, however, significant uncertainty still surrounds its measurement. In the U.S., in particular, two often-divergent GDP estimates exist, a widely-used expenditure-side version, $G D P_{E}$, and a much less widely-used income-side version, $G D P_{I}{ }^{1}$ Nalewaik (2010) and Fixler and Nalewaik (2009) make clear that, at the very least, GDP $P_{I}$ deserves serious attention and may even have properties in certain respects superior to those of $G D P_{E}$. That is, if forced to choose between $G D P_{E}$ and $G D P_{I}$, a surprisingly strong case exists for $G D P_{I}$. But of course one is not forced to choose between $G D P_{E}$ and $G D P_{I}$, and a GDP estimate based on both $G D P_{E}$ and $G D P_{I}$ may be superior to either one alone. In this paper we propose and implement a framework for obtaining such a blended estimate.

Our work is related to, and complements, Aruoba et al. (2012). There we took a forecasterror perspective, whereas here we take a measurement-error perspective. ${ }^{2}$ In particular, we work with a dynamic factor model in the tradition of Geweke (1977) and Sargent and Sims (1977), as used and extended by Watson and Engle (1983), Edwards and Howrey (1991), Harding and Scutella (1996), Jacobs and van Norden (2011), Kishor and Koenig (2011), and Fleischman and Roberts (2011), among others. ${ }^{3}$ That is, we view "true GDP" as a latent variable on which we have several indicators, the two most obvious being $G D P_{E}$ and $G D P_{I}$, and we then extract true GDP using optimal filtering techniques.

The measurement-error approach is time honored, intrinsically compelling, and very different from the forecast-combination perspective of Aruoba et al. (2012), for several reasons. ${ }^{4}$ First, it enables extraction of latent true GDP using a model with parameters estimated with exact likelihood or Bayesian methods, whereas the forecast-combination approach forces one to use calibrated parameters. Second, it delivers not only point extractions of latent true $G D P$ but also interval extractions, enabling us to assess the associated uncertainty. Third, the state-space framework in which the measurement-error models are embedded facilitates exploration of the relationship between GDP measurement errors and the economic environment, such as stage of the business cycle, which is of special interest. Fourth, the state-space

\footnotetext{
${ }^{1}$ Indeed we will focus on the U.S. because it is a key egregious example of unreconciled $G D P_{E}$ and $G D P_{I}$ estimates.

${ }^{2}$ Hence the pair of papers roughly parallels the well-known literature on "forecast error" and "measurement error" properties of data revisions; see Mankiw et al. (1984), Mankiw and Shapiro (1986), Faust et al. (2005), and Aruoba (2008).

${ }^{3}$ See also Smith et al. (1998), who take a different but related approach.

${ }^{4}$ On the time-honored aspect, see, for example, Gartaganis and Goldberger (1955).
} 
framework facilitates real-time analysis and forecasting, despite the fact that preliminary $G D P_{I}$ data are not available as quickly as those for $G D P_{E}$.

We proceed as follows. In section 2 we consider several measurement-error models and assess their identification status, which turns out to be challenging and interesting in the most realistic and hence compelling case. In section 3 we discuss the data, estimation framework and estimation results. In section 4 we explore the properties of our new GDP series. We conclude in section 5 .

\section{Five Measurement-Error Models of $G D P$}

We use dynamic-factor measurement-error models, which embed the idea that both $G D P_{E}$ and $G D P_{I}$ are noisy measures of latent true $G D P$. We work throughout with growth rates of $G D P_{E}, G D P_{I}$ and $G D P$ (hence, for example, $G D P_{E}$ denotes a growth rate). ${ }^{5}$ We assume throughout that true $G D P$ growth evolves with simple $A R(1)$ dynamics, and we entertain several measurement structures, to which we now turn.

\section{1 (Identified) 2-Equation Model: $\Sigma$ Diagonal}

Here we assume that the measurement errors are orthogonal to each other and to transition shocks at all leads and lags. The model has a natural state-space structure, and we write

$$
\begin{gathered}
{\left[\begin{array}{l}
G D P_{E t} \\
G D P_{I t}
\end{array}\right]=\left[\begin{array}{l}
1 \\
1
\end{array}\right] G D P_{t}+\left[\begin{array}{c}
\epsilon_{E t} \\
\epsilon_{I t}
\end{array}\right]} \\
G D P_{t}=\mu(1-\rho)+\rho G D P_{t-1}+\epsilon_{G t},
\end{gathered}
$$

where $G D P_{E t}$ and $G D P_{I t}$ are expenditure- and income-side estimates, respectively, $G D P_{t}$ is latent true $G D P$, and all shocks are Gaussian and uncorrelated at all leads and lags. That is, $\left(\epsilon_{G t}, \epsilon_{E t}, \epsilon_{I t}\right)^{\prime} \sim \operatorname{iid} N(\underline{0}, \Sigma)$, where

$$
\Sigma=\left[\begin{array}{ccc}
\sigma_{G G}^{2} & 0 & 0 \\
0 & \sigma_{E E}^{2} & 0 \\
0 & 0 & \sigma_{I I}^{2}
\end{array}\right] .
$$

\footnotetext{
${ }^{5}$ We will elaborate on the reasons for this choice later in section 3 .
} 
This model has been used countless times. As is well known, the Kalman filter delivers optimal extractions of $G D P_{t}$ conditional upon observed expenditure- and income-side measurements. Moreover, the model can be easily extended, and some of its restrictive assumptions relaxed, with no fundamental change. We now proceed to do so.

\section{2 (Identified) 2-Equation Model: $\Sigma$ Block-Diagonal}

The first extension is to allow for correlated measurement errors. This is surely important, as there is roughly a 25 percent overlap in the counts embedded in $G D P_{E}$ and $G D P_{I}$, and moreover, the same deflator is used for conversion from nominal to real magnitudes. ${ }^{6} \mathrm{We}$ write

$$
\begin{gathered}
{\left[\begin{array}{c}
G D P_{E t} \\
G D P_{I t}
\end{array}\right]=\left[\begin{array}{l}
1 \\
1
\end{array}\right] G D P_{t}+\left[\begin{array}{c}
\epsilon_{E t} \\
\epsilon_{I t}
\end{array}\right]} \\
G D P_{t}=\mu(1-\rho)+\rho G D P_{t-1}+\epsilon_{G t},
\end{gathered}
$$

where now $\epsilon_{E t}$ and $\epsilon_{I t}$ may be correlated contemporaneously but are uncorrelated at all other leads and lags, and all other definitions and assumptions are as before; in particular, $\epsilon_{G t}$ and $\left(\epsilon_{E t}, \epsilon_{I t}\right)^{\prime}$ are uncorrelated at all leads and lags. That is, $\left(\epsilon_{G t}, \epsilon_{E t}, \epsilon_{I t}\right)^{\prime} \sim i i d N(\underline{0}, \Sigma)$, where

$$
\Sigma=\left[\begin{array}{ccc}
\sigma_{G G}^{2} & 0 & 0 \\
0 & \sigma_{E E}^{2} & \sigma_{E I}^{2} \\
0 & \sigma_{I E}^{2} & \sigma_{I I}^{2}
\end{array}\right]
$$

Nothing is changed, and the Kalman filter retains its optimality properties.

\section{3 (Unidentified) 2-Equation Model, $\Sigma$ Unrestricted}

The second key extension is motivated by Fixler and Nalewaik (2009) and Nalewaik (2010), who document cyclicality in the statistical discrepancy $\left(G D P_{E}-G D P_{I}\right)$, which implies failure of the assumption that $\left(\epsilon_{E t}, \epsilon_{I t}\right)^{\prime}$ and $\epsilon_{G t}$ are uncorrelated at all leads and lags. Of particular concern is contemporaneous correlation between $\epsilon_{G t}$ and $\left(\epsilon_{E t}, \epsilon_{I t}\right)^{\prime}$. Hence we allow the measurement errors $\left(\epsilon_{E t}, \epsilon_{I t}\right)^{\prime}$ to be correlated with $G D P_{t}$, or more precisely, correlated

\footnotetext{
${ }^{6}$ See Aruoba et al. (2012) for more. Many of the areas of overlap are particularly poorly measured, such as imputed financial services, housing services, and government output.
} 
with $G D P_{t}$ innovations, $\epsilon_{G t}$. We write

$$
\begin{gathered}
{\left[\begin{array}{c}
G D P_{E t} \\
G D P_{I t}
\end{array}\right]=\left[\begin{array}{l}
1 \\
1
\end{array}\right] G D P_{t}+\left[\begin{array}{c}
\epsilon_{E t} \\
\epsilon_{I t}
\end{array}\right]} \\
G D P_{t}=\mu(1-\rho)+\rho G D P_{t-1}+\epsilon_{G t},
\end{gathered}
$$

where $\left(\epsilon_{G t}, \epsilon_{E t}, \epsilon_{I t}\right)^{\prime} \sim$ iid $N(\underline{0}, \Sigma)$, with

$$
\Sigma=\left[\begin{array}{ccc}
\sigma_{G G}^{2} & \sigma_{G E}^{2} & \sigma_{G I}^{2} \\
\sigma_{E G}^{2} & \sigma_{E E}^{2} & \sigma_{E I}^{2} \\
\sigma_{I G}^{2} & \sigma_{I E}^{2} & \sigma_{I I}^{2}
\end{array}\right] .
$$

In this environment the standard Kalman filter is rendered sub-optimal for extracting $G D P$, due to correlation between $\epsilon_{G t}$ and $\left(\epsilon_{E t}, \epsilon_{I t}\right)$, but appropriately-modified optimal filters are available.

Of course in what follows we will be concerned with estimating our measurement-equation models, so we will be concerned with identification. The diagonal- $\Sigma$ model (1)-(2) and the block-diagonal- $\Sigma$ model (3)-(4) are identified. Identification of less-restricted dynamic factor models, however, is a very delicate matter. In particular, it is not obvious that the unrestricted- $\Sigma$ model (5)-(6) is identified. Indeed it is not, as we prove in Appendix A. Hence we now proceed to determine minimal restrictions that achieve identification.

\section{4 (Identified) 2-Equation Model: $\Sigma$ Restricted}

The identification problem with the general model (5)-(6) stems from the fact that we can make true $G D P$ more volatile (increase $\sigma_{G G}^{2}$ ) and make the measurement errors more volatile (increase $\sigma_{E E}^{2}$ and $\sigma_{I I}^{2}$ ), but reduce the covariance between the fundamental shocks and the measurement errors (reduce $\sigma_{E G}^{2}$ and $\sigma_{I G}^{2}$ ), without changing the distribution of observables.

\subsubsection{Restricting the Original Parameterization}

But we can achieve identification by slightly restricting parameterization (5)-(6). In particular, as we show in Appendix A, the unrestricted system (5)-(6) is unidentified because the $\Sigma$ matrix has six free parameters with only five moment conditions to determine them. Hence we can achieve identification by restricting any single element of $\Sigma$. Imposing any such restriction would seem challenging, however, as we have no strong prior views directly 
on any single element of $\Sigma$. Fortunately, the problem is made tractable by a simple reparameterization.

\subsubsection{A Useful Re-Parameterization}

Define

$$
\zeta=\frac{\frac{1}{1-\rho^{2}} \sigma_{G G}^{2}}{\frac{1}{1-\rho^{2}} \sigma_{G G}^{2}+2 \sigma_{G E}^{2}+\sigma_{E E}^{2}} .
$$

Then, rather than fixing an element of $\Sigma$ to achieve identification, we can fix $\zeta$, about which we have a more natural prior view. In particular, at first pass we might take $\sigma_{G E}^{2} \approx 0$, in which case $0<\zeta<1$. Or, put differently, $\zeta>1$ would require a very negative $\sigma_{G E}^{2}$, which seems unlikely. All told, we view a $\zeta$ value less than, but close to, 1.0 as most natural. We take $\zeta=0.80$ as our benchmark in the empirical work that follows, although we explore a wide range of $\zeta$ values both below and above 1.0.

\section{5 (Identified) 3-Equation Model: $\Sigma$ Unrestricted}

Thus far we showed how to achieve identification by fixing a parameter, $\zeta$, and we noted that our prior is centered around $\zeta=0.80$. It is also of interest to know whether we can get some complementary data-based guidance on choice of $\zeta$. The answer turns out to be yes, by adding a third measurement equation with a certain structure.

Suppose, in particular, that we have an additional observable variable $U_{t}$ that loads on true $G D P_{t}$ with measurement error orthogonal to those of $G D P_{I}$ and $G D P_{E}$. In particular, consider the 3-equation model

$$
\begin{aligned}
{\left[\begin{array}{c}
G D P_{E t} \\
G D P_{I t} \\
U_{t}
\end{array}\right] } & =\left[\begin{array}{l}
0 \\
0 \\
\kappa
\end{array}\right]+\left[\begin{array}{l}
1 \\
1 \\
\lambda
\end{array}\right] G D P_{t}+\left[\begin{array}{c}
\epsilon_{E t} \\
\epsilon_{I t} \\
\epsilon_{U t}
\end{array}\right] \\
G D P_{t} & =\mu(1-\rho)+\rho G D P_{t-1}+\epsilon_{G t},
\end{aligned}
$$

where $\left(\epsilon_{G t}, \epsilon_{E t}, \epsilon_{I t}, \epsilon_{U t}\right)^{\prime} \sim \operatorname{iid} N(\underline{0}, \Omega)$, with

$$
\Omega=\left[\begin{array}{cccc}
\sigma_{G G}^{2} & \sigma_{G E}^{2} & \sigma_{G I}^{2} & \sigma_{G U}^{2} \\
\sigma_{E G}^{2} & \sigma_{E E}^{2} & \sigma_{E I}^{2} & 0 \\
\sigma_{I G}^{2} & \sigma_{I E}^{2} & \sigma_{I I}^{2} & 0 \\
\sigma_{U G}^{2} & 0 & 0 & \sigma_{U U}^{2}
\end{array}\right]
$$


Note that the upper-left 3x3 block of $\Omega$ is just $\Sigma$, which is now unrestricted. Nevertheless, as we prove in Appendix B, the 3-equation model (8)-(9) is identified. Of course some of the remaining elements of the overall $4 \mathrm{x} 4$ covariance matrix $\Omega$ are restricted, which is how we achieve identification in the 3-equation model, but the economically interesting sub-matrix, which the 3-equation model leaves completely unrestricted, is $\Sigma$.

Depending on the application, of course, it is not obvious that an identifying variable $U_{t}$ with measurement errors orthogonal to those of $G D P_{E}$ and $G D P_{I}$ (i.e., with stochastic properties that satisfy (9)), is available. Hence it is not obvious that estimation of the 3equation model (8)-(9) is feasible in practice, despite the model's appeal in principle. Indeed, much of the data collected from business surveys is used in the BEA's estimates, invalidating use of that data as $U_{t}$ since any measurement error in that data appears directly in either $G D P_{E}$ or $G D P_{I}$, producing correlation across the measurement errors. Moreover, variables drawn from business surveys similar to those used to produce $G D P_{E}$ and $G D P_{I}$, even if they are not used directly in the estimation of $G D P_{E}$ and $G D P_{I}$, might still be invalid identifying variables if the survey methodology itself produces similar measurement errors. ${ }^{7}$

Fortunately, however, some important macroeconomic data is collected not from surveys of businesses, but from samples of households. A sample of data drawn from a universe of households seems likely to have measurement errors that are different than those contaminating a data sample drawn from a universe of businesses, especially when the "universes" of businesses and households are not complete census counts, as is the case here. For example, the universe of business surveys is derived from tax records, so businesses not paying taxes will not appear on that list, but individuals working at that business may appear in the universe of households.

Importantly, very little data collected from household surveys are used to construct $G D P_{E}$ and $G D P_{I}$, so a $U_{t}$ variable computed from a household survey seems most likely to meet our identification conditions. The change in the unemployment rate is a natural choice (hence our notational choice $U_{t}$ ). $U_{t}$ arguably loads on true $G D P$ with a measurement error orthogonal to those of $G D P_{E}$ and $G D P_{I}$, because the $U_{t}$ data is being produced independently (by the BLS rather than the BEA) from different types of surveys. In addition, virtually all of the $G D P_{E}$ and $G D P_{I}$ data are estimated in nominal dollars and then converted to real dollars using a price deflator, whereas $U_{t}$ is estimated directly with no deflation.

\footnotetext{
${ }^{7}$ For example, if the business surveys used to produce $G D P_{E}$ and $G D P_{I}$ tend to oversample large firms, variables drawn from a business survey that also oversamples large firms may have measurement errors that are correlated with those in $G D P_{E}$ and $G D P_{I}$, absent appropriate corrections.
} 
Figure 1: Divergence Between $\hat{\Sigma}_{\zeta}$ and $\hat{\Sigma}_{3}$

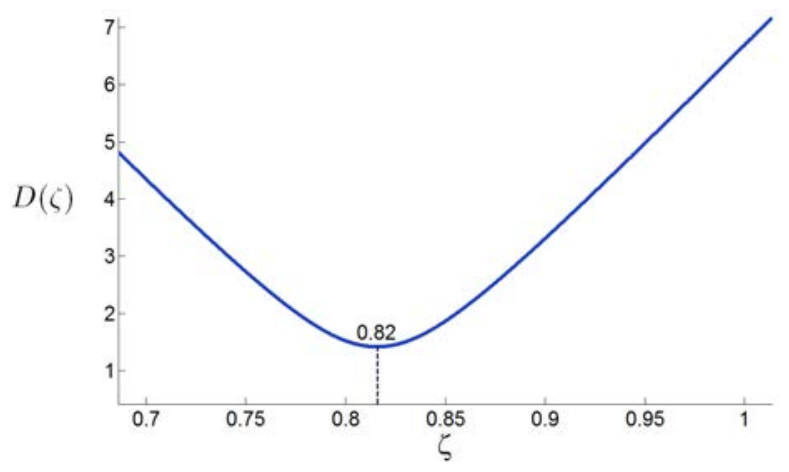

Notes: We show the Frobenius-norm divergence $D(\zeta)$ between $\widehat{\Sigma}_{\zeta}$ and $\widehat{\Sigma}_{3}$ as a function of $\zeta$. The optimum is $\zeta=0.82$. See text for details.

All told,we view "3-equation identification" as a useful complement to the " $\zeta$-identification" discussed earlier in section 2.4. All identifications involve assumptions. $\zeta$-identification involves introspection about likely values of $\zeta$, given its structure and components, and that introspection is of course subject to error. 3-equation identification involves introspection about various measurement-error correlations involving the newly-introduced third variable, which is of course also subject to error. Indeed the two approaches to identification are usefully used in tandem, and compared.

One can even view the 3 -equation approach as a device for implicitly selecting $\zeta$. In particular, we can find the $\zeta$ implied by the 3-equation model estimate, that is, find the $\zeta$ that minimizes the divergence between $\hat{\Sigma}_{\zeta}$ and $\hat{\Sigma}_{3}$, in an obvious notation. ${ }^{8}$ For example, using the Frobenius matrix-norm to measure divergence, we obtain an optimum of $\zeta^{*}=0.82$. We show the full surface in Figure 1, and the minimum is sharp and unique. The implied $\zeta^{*}$ of 0.82 is of course quite close to the directly-assessed value of 0.80 at which we arrived earlier, which lends additional credibility to the earlier assessment.

\section{Data and Estimation}

We intentionally work with a stationary system in growth rates, because we believe that measurement errors are best modeled as iid in growth rates rather than in levels, due to

\footnotetext{
${ }^{8}$ We will discuss subsequently the estimation procedure used to obtain $\hat{\Sigma}_{\zeta}$ and $\hat{\Sigma}_{3}$.
} 
Figure 2: GDP and Unemployment Data
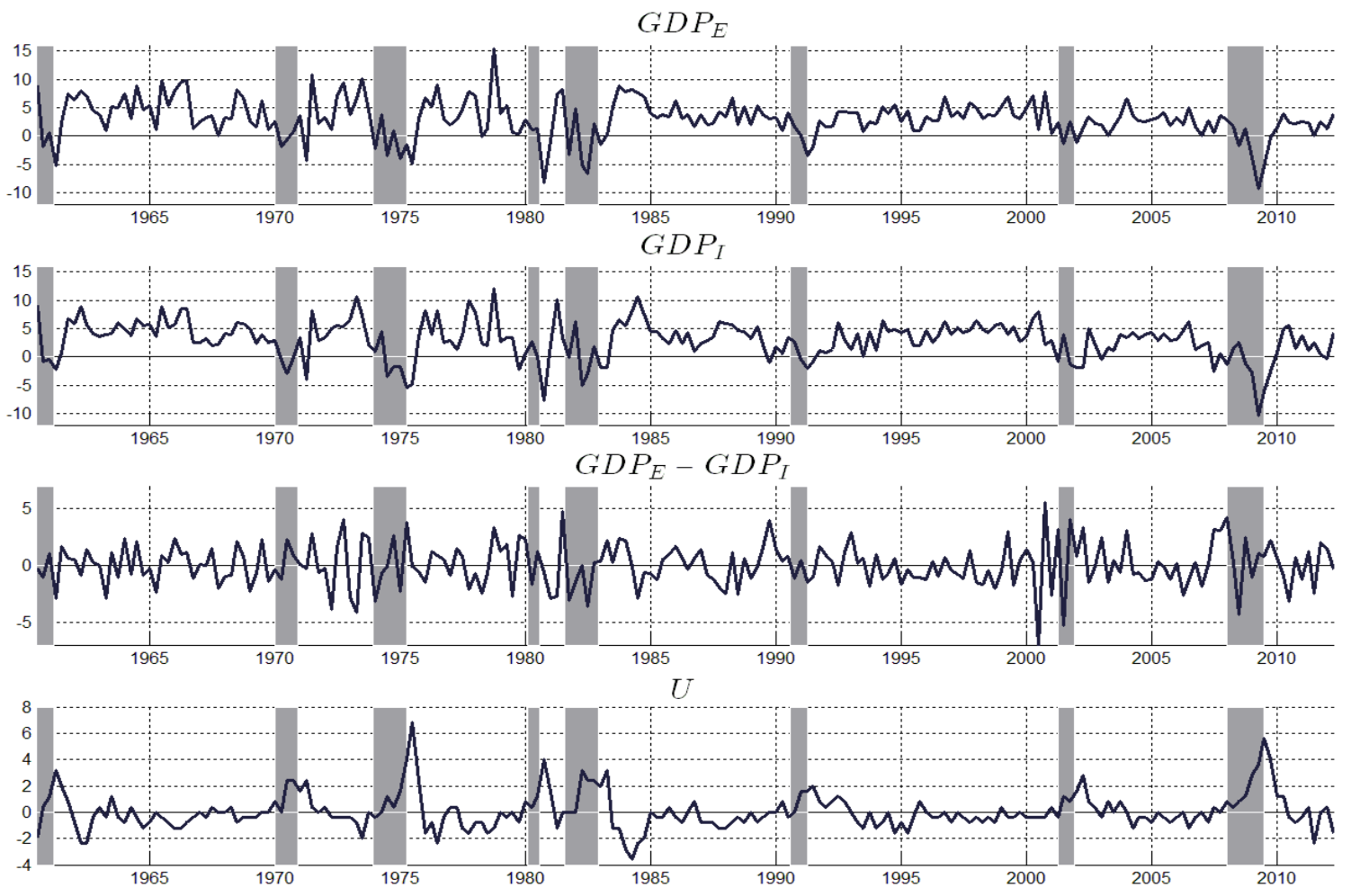

Notes: $G D P_{E}$ and $G D P_{I}$ are in growth rates and $U_{t}$ is in changes. All are measured in annualized percent.

BEA's devoting maximal attention to estimating the "best change." 9 In its above-cited "Concepts and Methods ..." document, for example, the BEA emphasizes that:

Best change provides the most accurate measure of the period-to-period movement in an economic statistic using the best available source data. In an annual revision of the NIPAs, data from the annual surveys of manufacturing and trade are generally incorporated into the estimates on a best-change basis. In the current quarterly estimates, most of the components are estimated on a best-change basis from the annual levels established at the most recent annual revision.

The monthly source data used to estimate $G D P_{E}$ (such as retail sales) and $G D P_{I}$ (such as nonfarm payroll employment) are generally produced on a best-change basis as well, using a so-called "link-relative estimator." This estimator computes growth rates using firms in the

\footnotetext{
${ }^{9}$ For example, see "Concepts and Methods in the U.S. National Income and Product Accounts," available at http://www. bea.gov/national/pdf/methodology/chapters1-4.pdf.
} 
sample in both the current and previous months, in contrast to a best-level estimator, which would generally use all the firms in the sample in the current month regardless of whether or not they were in the sample in the previous month. For example, for retail sales the BEA notes that: ${ }^{10}$

Advance sales estimates for the most detailed industries are computed using a type of ratio estimator known as the link-relative estimator. For each detailed industry, we compute a ratio of current-to-previous month weighted sales using data from units for which we have obtained usable responses for both the current and previous month.

Indeed the BEA produces estimates on a best-level basis only at 5-year benchmarks. These best-level benchmark revisions should drive only the very-low frequency variation in $G D P_{E}$, and thus probably matter very little for the quarterly growth rates estimated on a bestchange basis.

\subsection{Descriptive Statistics}

We show time-series plots of the "raw" $G D P_{E}$ and $G D P_{I}$ data in Figure 2, and we show summary statistics in the top panel of Table 1. Not captured in the table but also true is that the raw data are highly correlated; the simple correlations are $\operatorname{corr}\left(G D P_{E}, G D P_{I}\right)=0.85$, $\operatorname{corr}\left(G D P_{E}, U\right)=-0.67$, and $\operatorname{corr}\left(G D P_{I}, U\right)=-0.73$. Median $G D P_{I}$ growth is a bit higher than that of $G D P_{E}$, and $G D P_{I}$ growth is noticeably more persistent than that of $G D P_{E}$. Related, $G D P_{I}$ also has smaller $A R(1)$ innovation variance and greater predictability as measured by the predictive $R^{2}{ }^{11}$

\subsection{Bayesian Analysis of Measurement-Error Models}

Here we describe Bayesian analysis of our three-equation model, which of course also includes our various two-equation models as special cases. Bayesian estimation involves parameter estimation and latent state smoothing. First, we generate draws from the posterior distribution of the model parameters using a Random-Walk Metropolis-Hastings algorithm. Next, we apply a simulation smoother as described in Durbin and Koopman (2001) to obtain draws of the latent states conditional on the parameters.

\footnotetext{
${ }^{10}$ See http://www. census.gov/retail/marts/how_surveys_are_collected.html.

${ }^{11} \mathrm{On}$ this and related predictability measures, see Diebold and Kilian (2001).
} 
Table 1: Descriptive Statistics for Various GDP Series

\begin{tabular}{lcccccccccccc}
\hline & $\bar{x}$ & $50 \%$ & $\hat{\sigma}$ & $S k$ & $\hat{\rho}_{1}$ & $\hat{\rho}_{2}$ & $\hat{\rho}_{3}$ & $\hat{\rho}_{4}$ & $Q_{12}$ & $\hat{\sigma}_{e}$ & $R^{2}$ & $\hat{V}_{e}$ \\
\hline$G D P_{E}$ & 3.03 & 3.04 & 3.49 & -0.31 & .33 & .27 & .08 & .09 & 47.07 & 3.28 & .06 & 12.12 \\
$G D P_{I}$ & 3.02 & 3.39 & 3.40 & -0.55 & .47 & .27 & .22 & .08 & 81.60 & 2.99 & .12 & 11.43 \\
\hline$G D P_{M}$ 2-eqn, $\Sigma$ diag & 3.02 & 3.22 & 3.00 & -0.56 & .56 & .34 & .21 & .09 & 108.25 & 2.48 & .18 & 8.92 \\
$G D P_{M}$ 2-eqn, $\Sigma$ block & 3.02 & 3.35 & 2.64 & -0.64 & .70 & .45 & .28 & .13 & 170.08 & 1.89 & .29 & 6.90 \\
$G D P_{M}$ 2-eqn, $\zeta=0.65$ & 3.02 & 3.32 & 2.61 & -0.64 & .67 & .43 & .27 & .12 & 157.56 & 1.92 & .26 & 6.73 \\
$G D P_{M}$ 2-eqn, $\zeta=0.75$ & 3.02 & 3.30 & 2.77 & -0.63 & .65 & .41 & .26 & .11 & 148.23 & 2.08 & .25 & 7.60 \\
$G D P_{M}$ 2-eqn, $\zeta=0.80$ & 3.02 & 3.29 & 2.87 & -0.62 & .64 & .39 & .25 & .11 & 141.14 & 2.19 & .24 & 8.16 \\
$G D P_{M}$ 2-eqn, $\zeta=0.85$ & 3.02 & 3.31 & 2.89 & -0.64 & .66 & .41 & .28 & .12 & 153.27 & 2.15 & .25 & 8.29 \\
$G D P_{M}$ 2-eqn, $\zeta=0.95$ & 3.02 & 3.26 & 3.02 & -0.64 & .66 & .40 & .28 & .12 & 149.61 & 2.27 & .25 & 9.07 \\
$G D P_{M}$ 2-eqn, $\zeta=1.05$ & 3.01 & 3.22 & 3.12 & -0.65 & .67 & .40 & .28 & .12 & 155.60 & 2.30 & .26 & 9.69 \\
$G D P_{M}$ 2-eqn, $\zeta=1.15$ & 3.04 & 3.34 & 3.07 & -0.67 & .76 & .47 & .31 & .15 & 201.15 & 1.99 & .35 & 9.46 \\
$G D P_{M}$ 3-eqn & 3.02 & 3.37 & 3.02 & -1.14 & .63 & .37 & .21 & .03 & 141.79 & 2.33 & .23 & 9.03 \\
\hline$G D P_{F}$ & 3.02 & 3.29 & 3.30 & -0.51 & .46 & .29 & .19 & .07 & 78.28 & 2.92 & .12 & 10.80 \\
\hline
\end{tabular}

Notes: The sample period is 1960Q1-2011Q4. In the top panel we show statistics for the raw data. In the middle panel we show statistics for various posterior-median measurement-error-based (" $M$ ") estimates of true $G D P$, where all estimates are smoothed extractions. In the bottom panel we show statistics for the forecast-error-based ("F") estimate of true GDP produced by Aruoba et al. (2012). $\bar{x}, 50 \%, \hat{\sigma}$ and $S k$ are sample mean, median, standard deviation and skewness, respectively, and $\hat{\rho}_{\tau}$ is a sample autocorrelation at a displacement of $\tau$ quarters. $Q_{12}$ is the Ljung-Box serial correlation test statistic calculated using $\hat{\rho}_{1}, \ldots, \hat{\rho}_{12}$. $R^{2}=1-\frac{\hat{\sigma}_{e}^{2}}{\hat{\sigma}^{2}}$, where $\hat{\sigma}_{e}$ denotes the estimated disturbance standard deviation from a fitted $A R(1)$ model, is a predictive $R^{2} . \hat{V}_{e}$ is the unconditional variance implied by a fitted $A R_{1}$ model, $\hat{V}_{e}=\frac{\hat{\sigma}_{e}^{2}}{1-\hat{\rho}^{2}}$.

\subsubsection{State-Space Representation}

We proceed by introducing a state-space representation of (8) for estimation. Let $y_{t}=$ $\left[G D P_{E t}, G D P_{I t}, U_{t}\right]^{\prime}, C=[0,0, \kappa]^{\prime}, s_{t}=\left[G D P_{t}, \epsilon_{E t}, \epsilon_{I t}, \epsilon_{U t}\right]^{\prime}, D=[\mu(1-\rho), 0,0,0]^{\prime}, \epsilon_{t}=$ $\left[\epsilon_{G t}, \epsilon_{E t}, \epsilon_{I t}, \epsilon_{U t}\right]^{\prime}$ and

$$
Z=\left[\begin{array}{llll}
1 & 1 & 0 & 0 \\
1 & 0 & 1 & 0 \\
\lambda & 0 & 0 & 1
\end{array}\right], \quad \Phi=\left[\begin{array}{llll}
\rho & 0 & 0 & 0 \\
0 & 0 & 0 & 0 \\
0 & 0 & 0 & 0 \\
0 & 0 & 0 & 0
\end{array}\right]
$$

Our state-space model is

$$
\begin{gathered}
y_{t}=C+Z s_{t} \\
s_{t}=D+\Phi s_{t-1}+\epsilon_{t}, \quad \epsilon_{t} \sim N(0, \Omega) .
\end{gathered}
$$


We collect the parameters in (10) in $\Theta=\left(\mu, \rho, \sigma_{G G}^{2}, \sigma_{G E}^{2}, \sigma_{G I}^{2}, \sigma_{E E}^{2}, \sigma_{E I}^{2}, \sigma_{I I}^{2}, \sigma_{G U}^{2}, \sigma_{U U}^{2}, \kappa, \lambda\right)$.

\subsubsection{Metropolis-Hastings MCMC Algorithm}

Now let us proceed to our implementation of the Metropolis-Hastings MCMC Algorithm. Denote the number of MCMC draws by $N$. We first maximize the posterior density

$$
p\left(\Theta \mid Y_{1: T}\right) \propto p\left(Y_{1: T} \mid \Theta\right) p(\Theta)
$$

to obtain the mode $\Theta^{0}$ and construct a covariance matrix for the proposal density, $\Sigma_{\Theta}$, from the inverse Hessian of the log posterior density evaluated at $\Theta^{0}$. We also use $\Theta^{0}$ to initialize the algorithm. At each iteration $j$ we draw a proposed parameter vector $\Theta^{*} \sim N\left(\Theta^{j-1}, c \Sigma_{\Theta}\right)$, where $c$ is a scalar tuning parameter that we calibrate to achieve an acceptance rate of 25$30 \%$. We accept the proposed parameter vector, that is, we set $\Theta^{j}=\Theta^{*}$, with probability $\min \left\{1, \frac{p\left(Y_{1: T} \mid \Theta^{*}\right) p\left(\Theta^{*}\right)}{p\left(Y_{1: T} \Theta^{j-1}\right) p\left(\Theta^{j-1}\right)}\right\}$, and set $\Theta^{j}=\Theta^{j-1}$ otherwise. We adopt the convention that $p\left(\Theta^{*}\right)=0$ if the covariance matrix $\Omega$ implied by $\Theta^{*}$ is not positive definite. The results reported subsequently are based on $N=50,000$ iterations of the algorithm. We discard the first 25,000 draws and use the remaining draws to compute summary statistics for the posterior distribution.

\subsubsection{Filtering and Smoothing}

The evaluation of the likelihood function $p\left(Y_{1: T} \mid \Theta\right)$ requires the use of the Kalman filter. The Kalman filter recursions take the following form. Suppose that

$$
s_{t-1} \mid\left(Y_{1: t-1}, \Theta\right) \sim N\left(s_{t-1 \mid t-1}, P_{t-1 \mid t-1}\right)
$$

where $s_{t-1 \mid t-1}$ and $P_{t-1 \mid t-1}$ are the mean and variance of the latent state at $t-1$. Then the means and variances of the predictive densities $p\left(s_{t} \mid Y_{1: t-1}, \Theta\right)$ and $p\left(y_{t} \mid Y_{1: t-1}, \Theta\right)$ are

$$
\begin{aligned}
& s_{t \mid t-1}=D+\Phi s_{t-1 \mid t-1}, \quad P_{t \mid t-1}=\Phi P_{t-1 \mid t-1} \Phi^{\prime}+\Omega \\
& y_{t \mid t-1}=C+Z s_{t \mid t-1}, \quad F_{t \mid t-1}=Z P_{t \mid t-1} Z^{\prime},
\end{aligned}
$$


respectively. The contribution of observation $y_{t}$ to the likelihood function $p\left(Y_{1: T} \mid \Theta\right)$ is given by $p\left(y_{t} \mid Y_{1: t-1}, \Theta\right)$. Finally, the updating equations are

$$
\begin{aligned}
s_{t \mid t} & =s_{t \mid t-1}+\left(Z P_{t \mid t-1}\right)^{\prime} F_{t \mid t-1}^{-1}\left(y_{t}-\hat{y}_{t \mid t-1}\right) \\
P_{t \mid t} & =P_{t \mid t-1}-\left(Z P_{t \mid t-1}\right)^{\prime}\left(Z P_{t \mid t-1} Z^{\prime}\right)^{-1}\left(Z P_{t \mid t-1}\right),
\end{aligned}
$$

leading to

$$
s_{t} \mid\left(Y_{1: t}, \Theta\right) \sim N\left(s_{t \mid t}, P_{t \mid t}\right) .
$$

We initialize the Kalman filter by drawing $s_{0 \mid 0}$ from a mean-zero Gaussian stationary distribution whose covariance matrix, $P_{0 \mid 0}$, is the solution of the underlying Ricatti equation.

Because we are interested in inference for the latent $G D P$, we use the backward-smoothing algorithm of Carter and Kohn (1994) to generate draws recursively from $s_{t} \mid\left(S_{t+1: T}, Y_{1: T}, \Theta\right)$, $t=T-1, T-2, \ldots, 1$, where the last iteration of the Kalman filter recursion provides the initialization for the backward simulation smoother,

$$
\begin{array}{r}
s_{t \mid t+1}=s_{t \mid t}+P_{t \mid t} \Phi^{\prime} P_{t+1 \mid t}^{-1}\left(s_{t+1}-D-\Phi s_{t \mid t}\right) \\
P_{t \mid t+1}=P_{t \mid t}-P_{t \mid t} \Phi^{\prime} P_{t+1 \mid t}^{-1} \Phi P_{t \mid t} \\
\text { draw } s_{t} \mid\left(S_{t+1: T}, Y_{1: T}, \Theta\right) \sim N\left(s_{t \mid t+1}, P_{t \mid t+1}\right),
\end{array}
$$

$t=T-1, T-2, \ldots, 1$.

\subsection{Parameter Estimation Results}

Here we present and discuss estimation results for our various models. In Table 2 we show details of parameter prior and posterior distributions, as well as statistics describing the overall posterior and likelihood, for various 2-equation models, and in Table 3 we provide the same information for the 3-equation model.

The complete estimation information in the tables can be difficult to absorb fully, however, so here we briefly present aspects of the results in a more revealing way. For the 2-equation models, the parameters to be estimated are those in the transition equation and those in the covariance matrix $\Sigma$, which includes variances and covariances of both transition and measurement shocks. Hence we simply display the estimated transition equation and the estimated $\Sigma$ matrices. For the 3-equation model, we also need to estimate a factor loading in the measurement equation, so we display the estimated measurement equation as 
well. Below each posterior median parameter estimate, we show the posterior interquartile range in brackets.

For the 2-equation model with $\Sigma$ diagonal, we have

$$
\begin{aligned}
G D P_{t} & =\underset{[2.81,3.33]}{3.07}(1-0.53)+\underset{[0.48,0.57]}{0.53} G D P_{t-1}+\epsilon_{G t}, \\
\Sigma & =\left[\begin{array}{ccc}
6.90 & 0 & 0 \\
6.39,7.44] & & \\
0 & 2.32 & 0 \\
0 & 0 & 1.68 \\
0.12,2.55] & 01.52,1.85]
\end{array}\right] .
\end{aligned}
$$

For the 2-equation model with $\Sigma$ block-diagonal, we have

$$
\begin{aligned}
G D P_{t} & =\underset{[2.77,3.34]}{3.06}(1-0.62)+\underset{[0.57,0.68]}{0.62} G D P_{t-1}+\epsilon_{G t}, \\
\Sigma & =\left[\begin{array}{ccc}
5.17 & 0 & 0 \\
{[4.39,5.95]} & & \\
0 & 3.86 & 1.43 \\
& {[3.34,4.48]} & {[0.96,1.95]} \\
0 & 1.43 & 2.70 \\
& {[0.96,1.95]} & {[2.25,3.22]}
\end{array}\right] .
\end{aligned}
$$

For the 2-equation model with benchmark $\zeta=0.80$, we have

$$
\begin{gathered}
G D P_{t}=\underset{[2.79,3.35]}{3.08}(1-0.57)+\underset{[0.51,0.62]}{0.57} G D P_{t-1}+\epsilon_{G t}, \\
\Sigma=\left[\begin{array}{ccc}
7.09 & -0.69 & -0.38 \\
{[6.54,7.70]} & {[-1.15,-0.29]} & {[-0.74,-0.04]} \\
-0.69 & 3.90 & 1.29 \\
{[-1.15,-0.29]} & {[3.14,4.77]} & {[0.80,1.85]} \\
-0.38 & 1.29 & 2.36 \\
{[-0.74,-0.04]} & {[0.80,1.85]} & {[1.98,2.82]}
\end{array}\right] .
\end{gathered}
$$


Table 2: Priors and Posteriors, 2-Equation Models, 1960Q1-2011Q4

\begin{tabular}{|c|c|c|c|c|c|c|c|}
\hline & \multirow{2}{*}{$\begin{array}{c}\text { Prior } \\
\text { (Mean,Std.Dev) }\end{array}$} & \multicolumn{3}{|c|}{$\begin{array}{l}\text { Diagonal } \\
\text { Posterior }\end{array}$} & \multicolumn{3}{|c|}{$\begin{array}{c}\text { Block Diagonal } \\
\text { Posterior }\end{array}$} \\
\hline & & $25 \%$ & $50 \%$ & $75 \%$ & $25 \%$ & $50 \%$ & $75 \%$ \\
\hline$\mu$ & $\mathrm{N}(3,10)$ & 2.81 & 3.07 & 3.33 & 2.77 & 3.06 & 3.34 \\
\hline$\rho$ & $\mathrm{N}(0.3,1)$ & 0.48 & 0.53 & 0.57 & 0.57 & 0.62 & 0.68 \\
\hline$\sigma_{G G}^{2}$ & $\mathrm{IG}(10,15)$ & 6.39 & 6.90 & 7.44 & 4.39 & 5.17 & 5.95 \\
\hline$\sigma_{G E}^{2}$ & $\mathrm{~N}(0,10)$ & - & - & - & - & - & - \\
\hline$\sigma_{G I}^{2}$ & $\mathrm{~N}(0,10)$ & - & - & - & - & - & - \\
\hline$\sigma_{E E}^{2}$ & $\mathrm{IG}(10,15)$ & 2.12 & 2.32 & 2.55 & 3.34 & 3.86 & 4.48 \\
\hline$\sigma_{E I}^{2}$ & $\mathrm{~N}(0,10)$ & - & - & - & 0.96 & 1.43 & 1.95 \\
\hline$\sigma_{I I}^{E I}$ & $\mathrm{IG}(10,15)$ & 1.52 & 1.68 & 1.85 & 2.25 & 2.70 & 3.22 \\
\hline posterior & - & -984.57 & -983.46 & -982.60 & -986.23 & -985.00 & -984.01 \\
\hline \multirow[t]{4}{*}{ likelihood } & - & -951.68 & -950.41 & -949.43 & -950.70 & -949.49 & -948.60 \\
\hline & & \multicolumn{3}{|c|}{$\zeta=0.75$} & \multicolumn{3}{|c|}{$\zeta=0.80$} \\
\hline & Prior & \multicolumn{3}{|c|}{ Posterior } & \multicolumn{3}{|c|}{ Posterior } \\
\hline & (Mean,Std.Dev) & $25 \%$ & $50 \%$ & $75 \%$ & $25 \%$ & $50 \%$ & $75 \%$ \\
\hline$\mu$ & $\mathrm{N}(3,10)$ & 2.75 & 3.03 & 3.31 & 2.79 & 3.08 & 3.35 \\
\hline$\rho$ & $\mathrm{N}(0.3,1)$ & 0.53 & 0.59 & 0.64 & 0.51 & 0.57 & 0.62 \\
\hline$\sigma_{G G}^{2}$ & $\mathrm{IG}(10,15)$ & 5.78 & 6.31 & 6.92 & 6.54 & 7.09 & 7.70 \\
\hline$\sigma_{G E}^{2}$ & $\mathrm{~N}(0,10)$ & -0.76 & -0.29 & 0.15 & -1.15 & -0.69 & -0.29 \\
\hline$\sigma_{G I}^{2}$ & $\mathrm{~N}(0,10)$ & -0.34 & 0.01 & 0.34 & -0.74 & -0.38 & -0.04 \\
\hline$\sigma_{E E}^{2}$ & $\mathrm{IG}(10,15)$ & 3.08 & 3.88 & 4.75 & 3.14 & 3.90 & 4.77 \\
\hline$\sigma_{E I}^{2}$ & $\mathrm{~N}(0,10)$ & 0.73 & 1.23 & 1.78 & 0.80 & 1.29 & 1.85 \\
\hline$\sigma_{I I}^{2}$ & $\operatorname{IG}(10,15)$ & 1.94 & 2.30 & 2.76 & 1.98 & 2.36 & 2.82 \\
\hline posterior & - & -982.50 & -980.99 & -979.87 & -982.48 & -981.05 & -979.91 \\
\hline \multirow[t]{4}{*}{ likelihood } & - & -950.93 & -949.55 & -948.40 & -950.85 & -949.44 & -948.41 \\
\hline & & \multirow{2}{*}{\multicolumn{3}{|c|}{$\begin{array}{l}\zeta=0.85 \\
\text { Posterior }\end{array}$}} & \multirow{2}{*}{\multicolumn{3}{|c|}{$\begin{array}{l}\zeta=0.95 \\
\text { Posterior }\end{array}$}} \\
\hline & Prior & & & & & & \\
\hline & (Mean,Std.Dev) & $25 \%$ & $50 \%$ & $75 \%$ & $25 \%$ & $50 \%$ & $75 \%$ \\
\hline$\mu$ & $\mathrm{N}(3,10)$ & 2.72 & 2.96 & 3.14 & 2.84 & 3.03 & 3.25 \\
\hline$\rho$ & $\mathrm{N}(0.3,1)$ & 0.51 & 0.56 & 0.60 & 0.49 & 0.54 & 0.60 \\
\hline$\sigma_{G G}^{2}$ & $\mathrm{IG}(10,15)$ & 6.67 & 7.19 & 7.76 & 7.69 & 8.43 & 9.28 \\
\hline$\sigma_{G E}^{2}$ & $\mathrm{~N}(0,10)$ & -2.17 & -1.98 & -1.77 & -2.88 & -2.73 & -2.50 \\
\hline$\sigma_{G I}^{2}$ & $\mathrm{~N}(0,10)$ & -0.97 & -0.80 & -0.53 & -1.99 & -1.58 & -1.22 \\
\hline$\sigma_{E E}^{2}$ & $\mathrm{IG}(10,15)$ & 5.36 & 5.79 & 6.28 & 5.64 & 6.10 & 6.39 \\
\hline$\sigma_{E I}^{2}$ & $\mathrm{~N}(0,10)$ & 2.04 & 2.33 & 2.63 & 2.43 & 2.64 & 2.93 \\
\hline$\sigma_{I I}^{2}$ & $\mathrm{IG}(10,15)$ & 2.36 & 2.65 & 3.04 & 2.45 & 3.22 & 3.81 \\
\hline posterior & - & -982.62 & -981.40 & -980.48 & -984.09 & -982.80 & -981.57 \\
\hline \multirow[t]{4}{*}{ likelihood } & - & -949.42 & -948.25 & -947.49 & -950.19 & -948.84 & -947.81 \\
\hline & & \multirow{2}{*}{\multicolumn{3}{|c|}{$\begin{array}{l}\zeta=1.05 \\
\text { Posterior }\end{array}$}} & \multirow{2}{*}{\multicolumn{3}{|c|}{$\begin{array}{l}\zeta=1.15 \\
\text { Posterior }\end{array}$}} \\
\hline & Prior & & & & & & \\
\hline & (Mean,Std.Dev) & $25 \%$ & $50 \%$ & $75 \%$ & $25 \%$ & $50 \%$ & $75 \%$ \\
\hline$\mu$ & $\mathrm{N}(3,10)$ & 2.85 & 3.07 & 3.33 & 2.55 & 2.89 & 3.21 \\
\hline$\rho$ & $\mathrm{N}(0.3,1)$ & 0.48 & 0.53 & 0.58 & 0.52 & 0.56 & 0.61 \\
\hline$\sigma_{G G}^{2}$ & $\operatorname{IG}(10,15)$ & 8.92 & 9.57 & 10.25 & 9.07 & 9.88 & 10.73 \\
\hline$\sigma_{G E}^{2}$ & $\mathrm{~N}(0,10)$ & -4.04 & -3.88 & -3.70 & -5.61 & -5.50 & -5.22 \\
\hline$\sigma_{G I}^{2}$ & $\mathrm{~N}(0,10)$ & -3.09 & -2.65 & -2.29 & -4.38 & -4.21 & -4.01 \\
\hline$\sigma_{E E}^{2}$ & $\mathrm{IG}(10,15)$ & 6.74 & 7.13 & 7.41 & 8.51 & 9.07 & 9.30 \\
\hline$\sigma_{E I}^{2}$ & $\mathrm{~N}(0,10)$ & 3.23 & 3.46 & 4.13 & 5.29 & 5.52 & 5.89 \\
\hline$\sigma_{I I}^{2}$ & $\operatorname{IG}(10,15)$ & 3.27 & 143.66 & 4.43 & 5.68 & 6.00 & 6.31 \\
\hline posterior & - & -984.89 & -983.63 & -982.49 & -988.63 & -987.18 & -986.32 \\
\hline likelihood & - & -949.31 & -948.30 & -947.53 & -949.82 & -948.51 & -947.67 \\
\hline
\end{tabular}


Table 3: Priors and Posteriors, 3-Equation Model, 1960Q1-2011Q4

\begin{tabular}{ccrrr}
\hline \hline Parameter & Prior & \multicolumn{3}{c}{ Posterior } \\
& (Mean, Std) & $25 \%$ & $50 \%$ & $75 \%$ \\
\hline$\mu$ & $\mathrm{N}(3,10)$ & 2.60 & 2.78 & 2.95 \\
$\rho$ & $\mathrm{N}(0.3,1)$ & 0.54 & 0.58 & 0.63 \\
$\sigma_{G G}^{2}$ & $\mathrm{IG}(10,15)$ & 6.73 & 6.96 & 7.35 \\
$\sigma_{G E}^{2}$ & $\mathrm{~N}(0,10)$ & -1.27 & -1.10 & -0.84 \\
$\sigma_{G I}^{2}$ & $\mathrm{~N}(0,10)$ & -1.03 & -0.82 & -0.59 \\
$\sigma_{E E}^{2}$ & $\mathrm{IG}(10,15)$ & 4.17 & 4.57 & 4.79 \\
$\sigma_{E I}^{2}$ & $\mathrm{~N}(0,10)$ & 1.70 & 1.95 & 2.12 \\
$\sigma_{I I}^{2}$ & $\mathrm{IG}(10,15)$ & 2.54 & 3.07 & 3.27 \\
$\sigma_{G U}^{2}$ & $\mathrm{~N}(0,10)$ & 1.27 & 1.46 & 1.66 \\
$\sigma_{U U}^{2}$ & $\mathrm{IG}(0.3,10)$ & 0.50 & 0.59 & 0.71 \\
$\kappa$ & $\mathrm{N}(0,10)$ & 1.53 & 1.62 & 1.71 \\
$\lambda$ & $\mathrm{N}(-0.5,10)$ & -0.55 & -0.52 & -0.50 \\
posterior & - & -1251.1 & -1249.6 & -1248.3 \\
likelihood & - & -1199.0 & -1197.5 & -1196.2 \\
\hline \hline
\end{tabular}

Finally, for the 3-equation model, we have

$$
\begin{gathered}
{\left[\begin{array}{c}
G D P_{E t} \\
G D P_{I t} \\
U_{t}
\end{array}\right]=\left[\begin{array}{c}
0 \\
0 \\
1.62 \\
{[1.53,1.71]}
\end{array}\right]+\left[\begin{array}{c}
1 \\
1 \\
-0.52 \\
{[-0.55,-0.50]}
\end{array}\right] G D P_{t}+\left[\begin{array}{c}
\epsilon_{E t} \\
\epsilon_{I t} \\
\epsilon_{U t}
\end{array}\right]} \\
G D P_{t}=\underset{[2.60,2.95]}{2.78}(1-0.58)+\underset{[0.54,0.63]}{0.58} G D P_{t-1}+\epsilon_{G t}, \\
{\left[\begin{array}{c}
\epsilon_{G t} \\
\epsilon_{E t} \\
\epsilon_{I t} \\
\epsilon_{U t}
\end{array}\right] \sim N\left(\left[\begin{array}{l}
0 \\
0 \\
0 \\
0
\end{array}\right],\left[\begin{array}{cccc}
6.96 & -1.10 & -0.82 & 1.46 \\
{[6.73,7.35]} & {[-1.27,-0.84]} & {[-1.03,-0.59]} & {[1.27,1.66]} \\
-1.10 & 4.57 & 1.95 & 0 \\
{[-1.27,-0.84]} & {[4.17,4.79]} & {[1.70,2.12]} & 0 \\
-0.82 & 1.95 & 3.07 & 0 \\
{[-1.03,-0.59]} & {[1.70,2.12]} & {[2.54,3.27]} & 0 \\
1.46 & 0 & 0 & 0.59 \\
{[1.27,1.66]} & 0 & 0.50,0.71]
\end{array}\right]\right)}
\end{gathered}
$$

Many aspects of the results are noteworthy; here we simply mention a few. First, every posterior interval in every model reported above excludes zero. Hence the diagonal and block 
Figure 3: GDP Sample Paths, 1960Q1-2011Q4
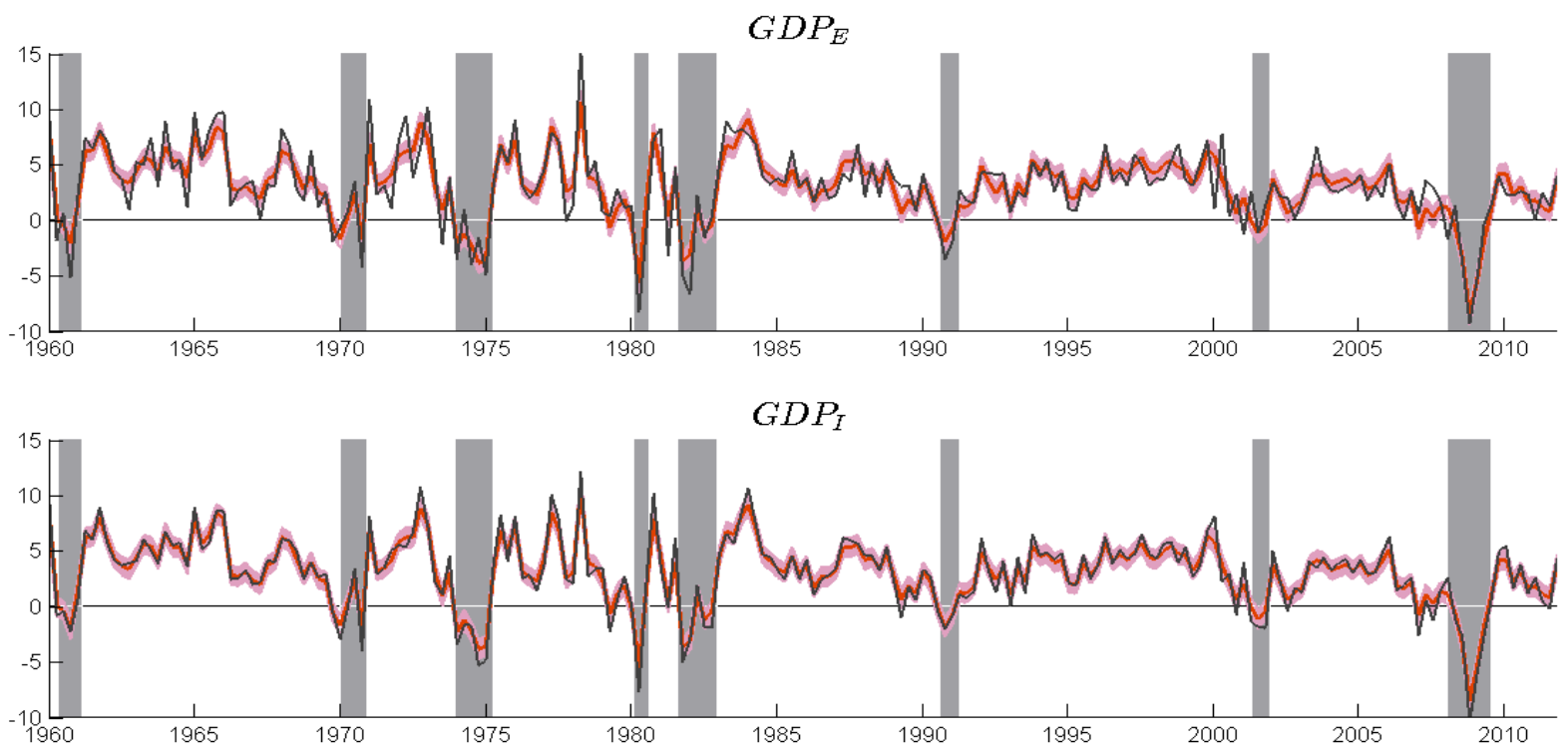

Notes: In each panel we show the sample path of $G D P_{M}$ in red together with a light-red posterior interquartile range, and we show one of the competitor series in black. For $G D P_{M}$ we use our benchmark estimate from the 2-equation model with $\zeta=0.80$.

diagonal models do not appear satisfactory.

Second, the $\Sigma$ estimates are qualitatively similar across specifications. Covariances are always negative, as per our conjecture based on the counter-cyclicality in the statistical discrepancy $\left(G D P_{E}-G D P_{I}\right)$ documented by Fixler and Nalewaik (2009) and Nalewaik (2010). Shock variances always satisfy $\hat{\sigma}_{G G}^{2}>\hat{\sigma}_{E E}^{2}>\hat{\sigma}_{I I}^{2}$.

Finally, $G D P_{M}$ is highly serially correlated across all specifications $(\rho \approx .6)$, much more so than the current "consensus" based on $G D P_{E}(\rho \approx .3)$. We shall have much more to say about these and other results in the next section.

\section{New Perspectives on the Properties of $G D P$}

Our various extracted $G D P_{M}$ series differ in fundamental ways from other measures, such as $G D P_{E}$ and $G D P_{I}$. Here we discuss some of the most important differences. 
Figure 4: GDP Sample Paths, 2007Q1-2009Q4
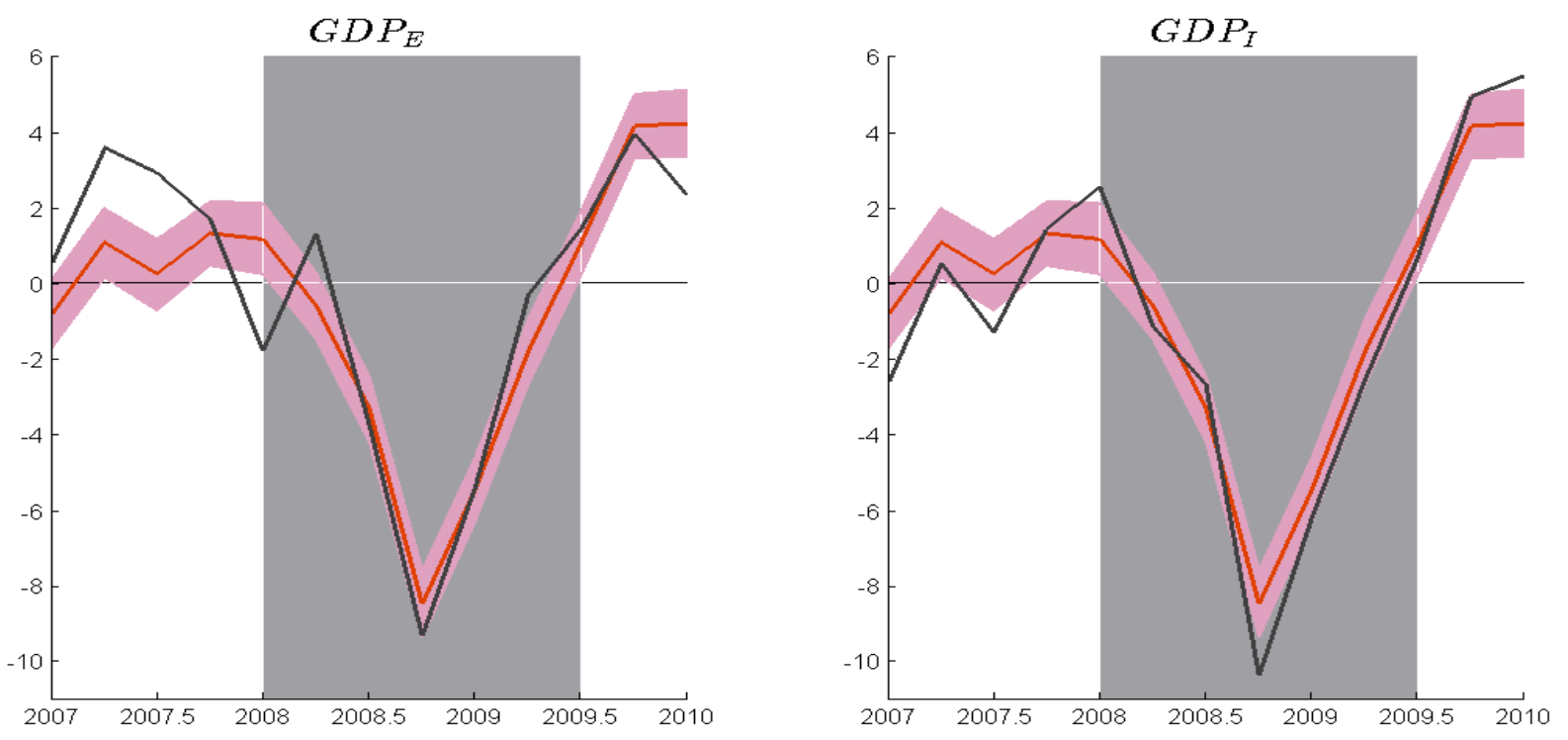

Notes: In each panel we show the sample path of $G D P_{M}$ in red together with a light-red posterior interquartile range, and we show one of the competitor series in black. For $G D P_{M}$ we use our benchmark estimate from the 2-equation model with $\zeta=0.80$.

\subsection{GDP Sample Paths}

Let us begin by highlighting the sample-path differences between our $G D P_{M}$ and the obvious competitors $G D P_{E}$ and $G D P_{I}$. We make those comparisons in Figure 3. In each panel we show the sample path of $G D P_{M}$ in red together with a light-red posterior interquartile range, and we show one of the competitor series in black. ${ }^{12}$ In the top panel we show $G D P_{M}$ vs. $G D P_{E}$. There are often wide divergences, with $G D P_{E}$ well outside the posterior interquartile range of $G D P_{M}$. Indeed $G D P_{E}$ is substantially more volatile than $G D P_{M}$. In the bottom panel of Figure 3 we show $G D P_{M}$ vs. $G D P_{I}$. Noticeable divergences again appear often, with $G D P_{I}$ also outside the posterior interquartile range of $G D P_{M}$. The divergences are not as pronounced, however, and the "excess volatility" apparent in $G D P_{E}$ is less apparent in $G D P_{I}$. That is because, as we will show later, $G D P_{M}$ loads relatively more heavily on $G D P_{I}$.

To emphasize the economic importance of the differences in competing real activity assessments, in Figure 4 we focus on the tumultuous period 2007Q1-2009Q4. The figure makes

\footnotetext{
${ }^{12}$ For $G D P_{M}$ we use our benchmark estimate from the 2-equation model with $\zeta=0.80$.
} 
clear not only that both $G D P_{E}$ and $G D P_{I}$ can diverge substantially from $G D P$, but also that the timing and nature of their divergences can be very different. In 2007Q3, for example, $G D P_{E}$ growth was strongly positive and $G D P_{I}$ growth was negative.

\subsection{Linear GDP Dynamics}

In our framework, population true $G D P_{t}$ is simply a pair $\left(\sigma_{G G}^{2}, \rho\right)$. In Figure 5 we show those pairs across MCMC draws for all of our measurement-error models, and for comparison we show $\left(\sigma^{2}, \rho\right)$ values corresponding to $A R(1)$ models fit to $G D P_{E}$ alone and $G D P_{I}$ alone. In addition, in Table 1 we show a variety of statistics quantifying the properties of our various $G D P_{M}$ measures vs. those of $G D P_{E}, G D P_{I}$ and $G D P_{F}$.

First consider Figure 5. Across measurement-error models $M, G D P_{M}$ is robustly more serially correlated than both $G D P_{E}$ and $G D P_{I}$, and it also has a smaller innovation variance. Hence most of our models achieve closely-matching unconditional variances, but they are composed of very different underlying $\left(\sigma^{2}, \rho\right)$ values from those corresponding to $G D P_{E}$. $G D P_{M}$ has smaller shock volatility, but much more shock persistence - roughly double that of $G D P_{E}$ ( $\rho$ of roughly 0.60 for $G D P_{M}$ vs. 0.30 for $G D P_{E}$ ).

Now consider Table 1. The various $G D P_{M}$ series are all less volatile than each of $G D P_{E}$, $G D P_{I}$ and $G D P_{F}$, and a bit more skewed left. Most noticeably, the $G D P_{M}$ series are much more strongly serially correlated than the $G D P_{E}, G D P_{I}$ and $G D P_{F}$ series, and with smaller innovation variances. This translates into much higher predictive $R^{2}$ 's for $G D P_{M}$. Indeed $G D P_{M}$ is twice as predictable as $G D P_{I}$ or $G D P_{F}$, which in turn are twice as predictable as $G D P_{E}$.

\subsection{Non-Linear $G D P$ Dynamics}

In Table 4 we show Markov-switching $A R(1)$ model results for a variety of $G D P$ series. The model allows for simultaneous switching in both mean and serial-correlation parameters. The model switches between high- and low-growth states, with low-growth states generally including recessions as defined by the National Bureau of Economic Research's Business Cycle Dating Committee (see also Nalewaik (2012)). The most interesting aspect of the results concerns the estimated low- and high-state serial-correlation parameters $\left(\hat{\rho}_{0}\right.$ and $\hat{\rho}_{1}$, respectively).

First, always and everywhere, $\hat{\rho}_{0}>\hat{\rho}_{1}$; that is, a disproportionate share of overall se-

rial correlation comes from low-growth states. This interesting result parallels recent work 
Figure 5: $\left(\hat{\rho}, \hat{\sigma}_{G G}^{2}\right)$ Pairs Across MCMC Draws
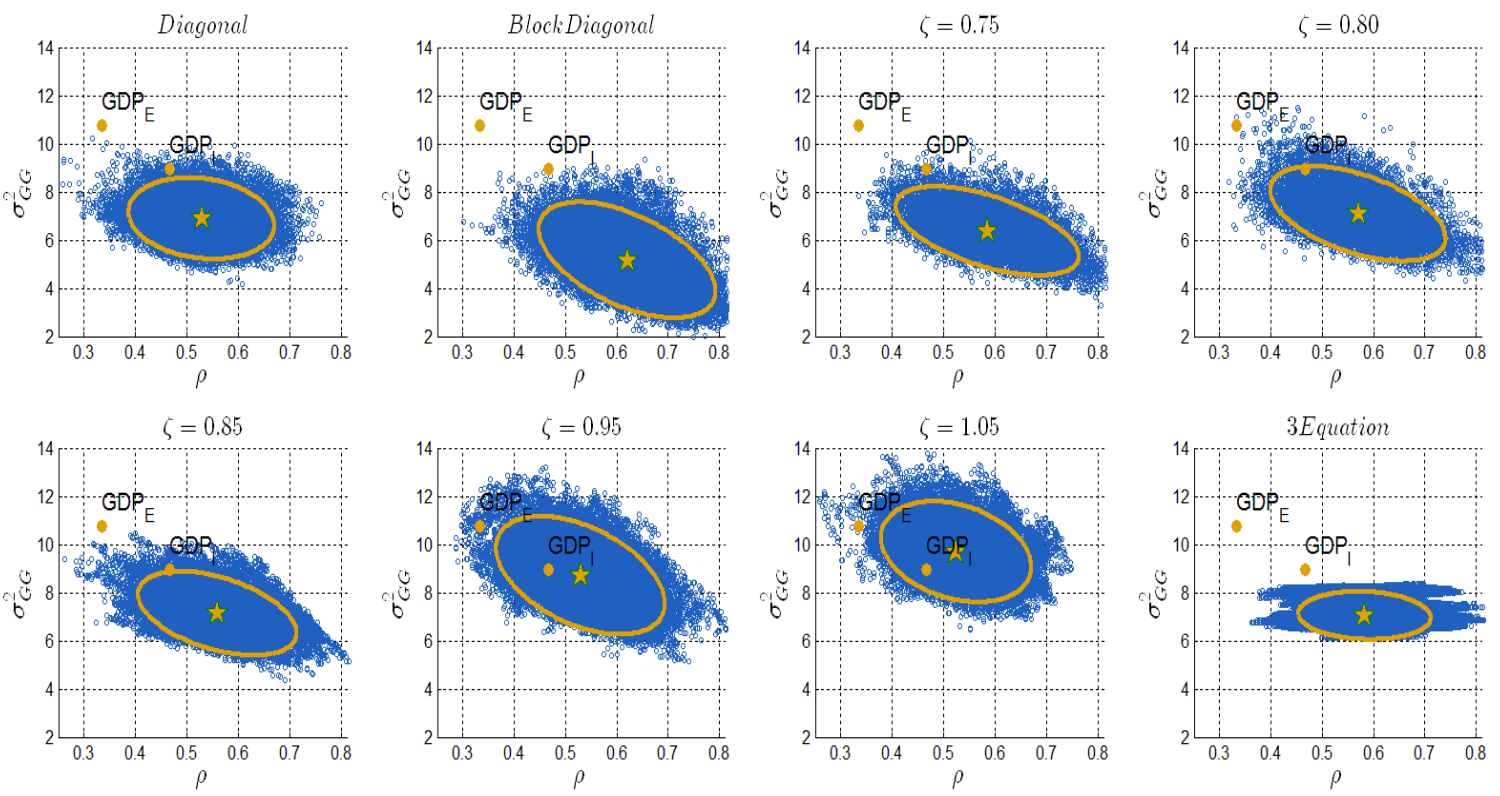

Notes: Solid lines indicate $90 \%\left(\sigma_{G G}^{2}, \rho\right)$ posterior coverage ellipsoids for the various models. Stars indicate posterior median values. The sample period is 1960Q1-2011.Q4. For comparison we show $\left(\sigma^{2}, \rho\right)$ values corresponding to $A R(1)$ models fit to $G D P_{E}$ alone and $G D P_{E}$ alone.

indicating that a disproportionate share of stock market return predictability comes from recessions (Rapach et al. (2010)), as well as work showing that shocks to business orders for capital goods are more persistent in downturns (Nalewaik and Pinto (2012)).

Second, comparison of $G D P_{I}$ to $G D P_{E}$ reveals that they have identical $\hat{\rho}_{0}$ values $(0.55)$, but that $\hat{\rho}_{1}$ is much bigger for $G D P_{I}$ than for $G D P_{E}$ (0.31 vs. 0.14). Hence the stronger overall serial correlation of $G D P_{I}$ comes entirely from its stronger serial correlation during expansions.

Finally, comparison of $G D P_{M}$ to $G D P_{E}$ reveals much bigger $\hat{\rho}_{0}$ and $\hat{\rho}_{1}$ values for $G D P_{M}$ than for $G D P_{E}$, regardless of the particular measurement-error model $M$ examined. The general finding of $\hat{\rho}_{0}>\hat{\rho}_{1}$ is preserved, but both $\hat{\rho}_{0}$ and $\hat{\rho}_{1}$ are much larger for $G D P_{M}$ than for $G D P_{E}$. In our benchmark 2-equation model with $\zeta=0.80$, for example, we have $\hat{\rho}_{0}=0.78$ and $\hat{\rho}_{1}=0.55$. 
Table 4: Regime-Switching Model Estimates, 1960Q1-2011Q4

\begin{tabular}{lcccccccc}
\hline & $\hat{\mu}_{0}$ & $\hat{\mu}_{1}$ & $\hat{\rho}_{0}$ & $\hat{\rho}_{1}$ & $\hat{\sigma}_{H}^{2}$ & $\hat{\sigma}_{L}^{2}$ & $\hat{p}_{00}$ & $\hat{p}_{11}$ \\
\hline$G D P_{E}$ & 1.31 & 4.71 & 0.55 & 0.14 & 16.55 & 4.81 & 0.81 & 0.88 \\
$G D P_{I}$ & 1.28 & 4.87 & 0.55 & 0.31 & 12.07 & 5.51 & 0.82 & 0.87 \\
\hline$G D P_{M}$ 2-eqn, $\Sigma$ diag & 1.76 & 5.12 & 0.73 & 0.41 & 9.81 & 3.37 & 0.83 & 0.85 \\
$G D P_{M}$ 2-eqn, $\Sigma$ block & 1.75 & 4.72 & 0.83 & 0.63 & 6.22 & 2.41 & 0.81 & 0.86 \\
$G D P_{M}$ 2-eqn, $\zeta=0.80$ & 1.79 & 4.95 & 0.78 & 0.55 & 7.96 & 3.04 & 0.82 & 0.85 \\
$G D P_{M}$ 3-eqn & 1.88 & 5.32 & 0.88 & 0.39 & 7.85 & 2.95 & 0.80 & 0.85 \\
\hline$G D P_{F}$ & 1.51 & 4.93 & 0.64 & 0.30 & 13.20 & 4.17 & 0.82 & 0.87 \\
\hline
\end{tabular}

Notes: In the top panel we show posterior median estimates for two-state regime-switching $A R(1)$ models fit to raw data. In the middle panel we show posterior median estimates for Regime-switching models fit to $G D P_{M}$, and in the bottom panel we show posterior median estimates for regime-switching models fit to $G D P_{F}$. We allow for a one-time structural break in volatility in 1984 (the "Great Moderation").

\subsection{On the Relative Contributions of $G D P_{E}$ and $G D P_{I}$ to $G D P_{M}$}

It is of interest to know how the observed indicators $G D P_{E}$ and $G D P_{I}$ contribute to our extracted true GDP. We do this in two ways; in section 4.4.1 we examine Kalman gains, and in section 4.4.2 we find the convex combination of $G D P_{E}$ and $G D P_{I}$ closest to our extracted $G D P$.

\subsubsection{Kalman Gains}

The Kalman gains associated with $G D P_{E}$ and $G D P_{I}$ govern the amount by which news about $G D P_{E}$ and $G D P_{I}$, respectively, causes the optimal extraction of $G D P_{t}$ (conditional on time- $t$ information) to differ from the earlier optimal prediction of $G D P_{t}$ (conditional on time- $(t-1)$ information). Put more simply, the Kalman gain of $G D P_{E}$ (resp. GDP $P_{I}$ measures its importance in influencing $G D P_{M}$, and hence in informing our views about latent true $G D P$.

We summarize the posterior distributions of Kalman gains in Figure 6. Posterior median $G D P_{I}$ Kalman gains are large in absolute terms, and most notably, very large relative to those for $G D P_{E}$. Indeed posterior median $G D P_{E}$ Kalman gains are zero in several specifications. In any event, it is clear that $G D P_{I}$ plays a larger role in informing us about $G D P$ than does $G D P_{E}$. For our benchmark $\zeta$-model with $\zeta=0.80$, the posterior median $G D P_{I}$ and $G D P_{E}$ Kalman gains are 0.59 and 0.23 , respectively. 
Figure 6: $\left(K G_{E}, K G_{I}\right)$ Pairs Across MCMC Draws
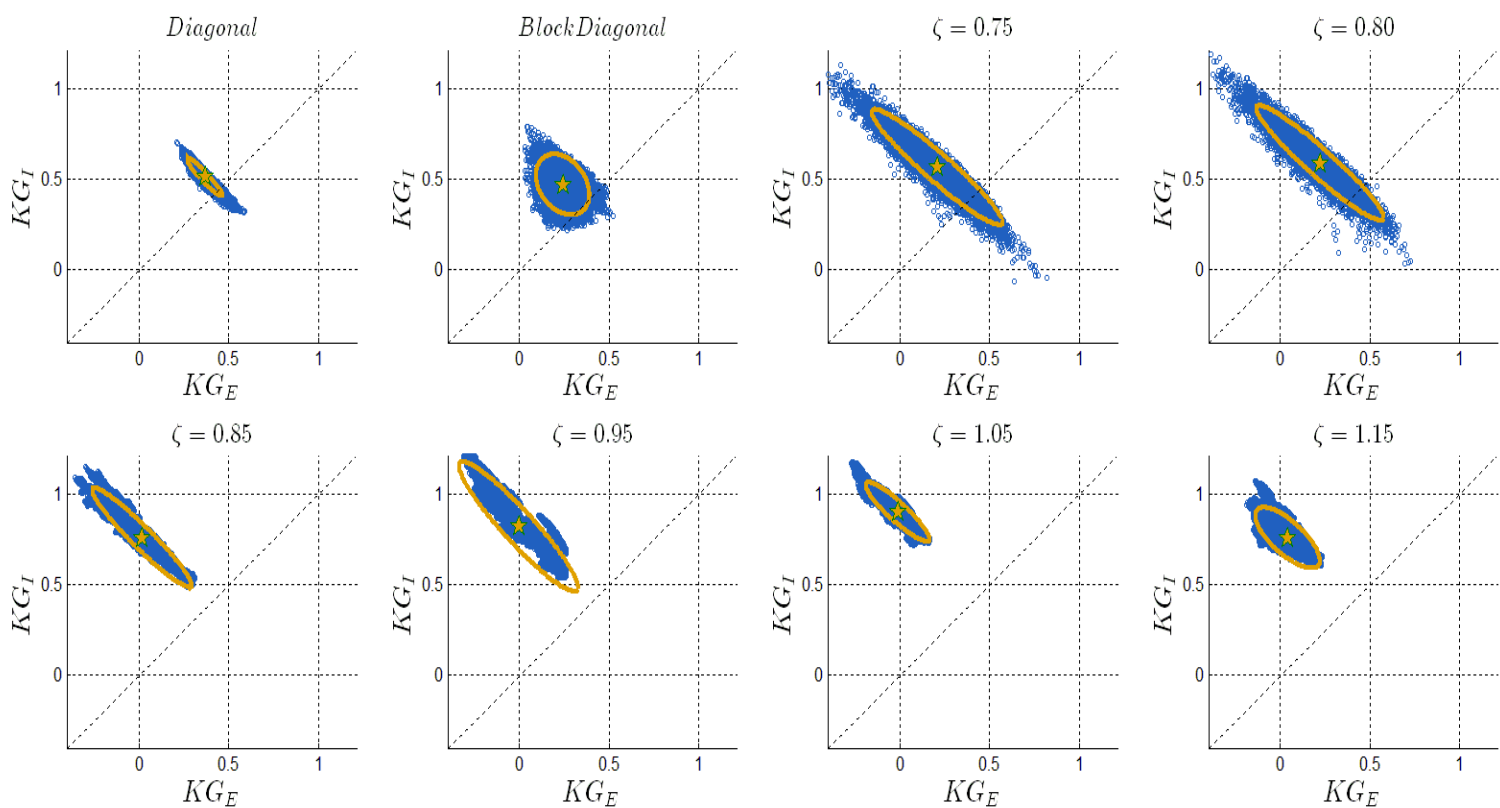

Notes: Solid lines indicate $90 \%$ posterior coverage ellipsoids. Stars indicate posterior median values.

\subsubsection{Closest Convex Combination}

The Kalman filter extractions average not only over space, but also over time. Nevertheless, we can ask what contemporaneous convex combination of $G D P_{E}$ and $G D P_{I}, \lambda G D P_{E}+(1-$ ג) $G D P_{I}$, is closest to the extracted $G D P_{M}$. That is, we can find $\lambda^{*}=\operatorname{argmin}_{\lambda} L(\lambda)$, where $L(\lambda)$ is a loss function. Under quadratic loss we have

$$
\lambda^{*}=\operatorname{argmin}_{\lambda} \sum_{t=1}^{T}\left[\left(\lambda G D P_{E t}+(1-\lambda) G D P_{I t}\right)-G D P_{M t}\right]^{2},
$$

where $G D P_{M t}$ is our smoothed extraction of true $G D P_{t}$. Over our sample of 1960Q12011Q4, the optimum under quadratic loss is $\lambda^{*}=0.29$. The minimum is quite sharp, as we show in Figure 7, and it puts more than twice as much weight on $G D P_{I}$ than on $G D P_{E}$. That weighting accords closely with both the Kalman gain results discussed above and the forecast-combination calibration results in Aruoba et al. (2012). It does not, of course, mean that time series of $G D P_{M}$ will "match" time series of $G D P_{F}$, because the Kalman filter does much more than simple contemporaneous averaging of $G D P_{E}$ and $G D P_{I}$ in its extraction of latent true $G D P$. 
Figure 7: Closest Convex Combination

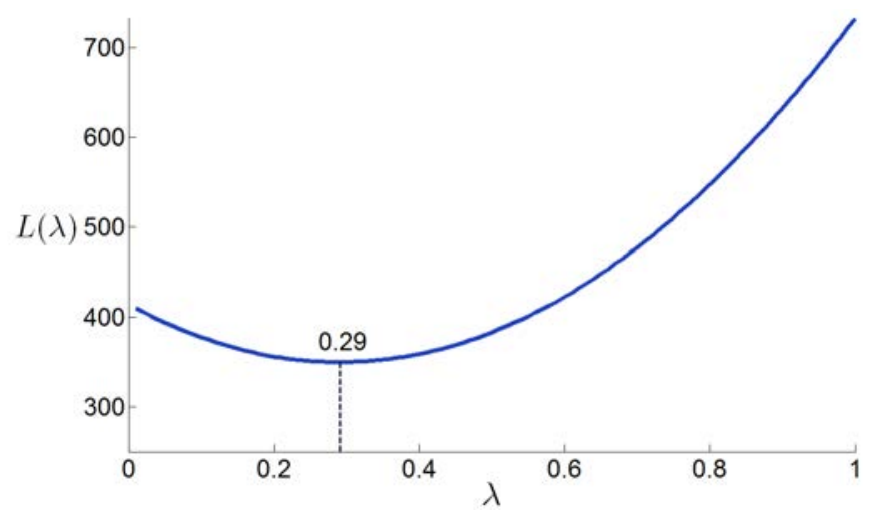

Notes: We show quadratic loss, $L(\lambda)=\sum_{t=1960 Q 1}^{2011 Q 4}\left[\left(\lambda G D P_{E t}+(1-\lambda) G D P_{I t}\right)-G D P_{M t}\right]^{2}$, as a function of $\lambda$. We obtain $G D P_{M t}$ from the 3-equation model.

\subsection{A Final Remark on the Serial Correlation in $G D P_{M}$}

Obviously a key result of our analysis is the strong serial correlation (persistence, forecastability, smoothness, ...) of our extracted $G D P_{M}$, regardless of the particular specification. One might perhaps wonder whether this is a spurious artifact of our extraction method, which effectively amounts to a Kalman "smoother." We hasten to add that the answer is no. Indeed optimal extractions of covariance stationary series (in our, case latent true GDP growth) must be less variable than the series being extracted, because the optimal extraction is a conditional expectation. ${ }^{13}$ Given our models, with Gaussian errors and under quadratic loss, any other GDP extractions are sub-optimal and hence inferior.

\section{Concluding Remarks and Directions for Future Re- search}

We produce several estimates of $G D P$ that blend both $G D P_{E}$ and $G D P_{I}$. All estimates feature $G D P_{I}$ prominently, and our blended $G D P$ estimate has properties quite different from those of the "traditional" $G D P_{E}$ (as well as $G D P_{I}$ ). In a sense we build on the literature on "balancing" the national income accounts, which extends back almost as far as

\footnotetext{
${ }^{13}$ The forecast-error approach of Aruoba et al. (2012) also has optimality properties, but of a different sort, and there is no reason why in the forecast-error framework the optimal combination should be smoother than latent true GDP growth. Instead it could go either way, depending on the correlation of the forecast errors in $G D P_{E}$ and $G D P_{I}$.
} 
national income accounting itself, as for example in Stone et al. (1942). We do not, however, advocate that the U.S. publish only $G D P_{M}$, as there may at times be value in being able to see the income and expenditure sides separately. But we would advocate the additional calculation of $G D P_{M}$ and using it as the benchmark $G D P$ estimate.

Many interesting extensions are possible, including (1) Allowing for richer GDP dynamics. We might want to add $G D P_{t-1}$ to the unemployment equation in addition to $G D P_{t}$, because unemployment tends to lag GDP; (2) Allowing for serially correlated unemployment measurement errors. We might want to allow the unemployment measurement errors to be serially correlated, because they are really more than just measurement errors, in contrast to the $G D P_{E}$ and $G D P_{I}$ measurement errors; (3) Including additional identifying variables, which could be used alternatively or in addition to unemployment. The Michigan consumer confidence index, for example, is released before $G D P_{E}$ and $G D P_{I}$, which are not based on it in any way. Another possibility is non-U.S. GDP.

Perhaps the two most interesting directions for future work, however, concern forecasting and real-time analysis. First consider forecasting. When forecasting a "traditional" GDP series such as $G D P_{E}$, we must take it as given (i.e., we must ignore measurement error). The analogous procedure in our framework would take $G D P_{M}$ as given, modeling and forecasting it directly, ignoring the fact that it is based on a first-stage extraction subject to error. Fortunately, however, in our framework we need not do that. Instead we can estimate and forecast directly from the dynamic factor model, accounting for all sources of uncertainty, which should translate into superior interval and density forecasts. Related, it would be interesting to calculate directly the point, interval and density forecast functions corresponding to the measurement-error model.

Second, consider real-time analysis. Although $G D P_{I}$ data are not as timely as $G D P_{E}$ data, our filtering framework still uses all available data efficiently, appropriately handling any missing data. A key insight is that when using simple convex combinations as in the forecast-error approach of Aruoba et al. (2012), missing $G D P_{I}$ data for the most-recent quarter(s) forces all weight to be put on $G D P_{E}$. Our measurement-error framework is very different, however, because the Kalman filter averages not just over space, but also over time, and earlier quarters for which we do have $G D P_{I}$ data are informative for the most-recent quarters with "missing" $G D P_{I}$ data. 


\section{Appendices}

Here we report various details of theory, establishing identification results for the two- and three-variable models in appendices $\mathrm{A}$ and $\mathrm{B}$, respectively. The identification analysis is based on Komunjer and $\mathrm{Ng}$ (2011).

\section{A Identification in the Two-Variable Model}

The constants in the state-space model can be identified from the means of $G D P_{E t}$ and $G D P_{I t}$. To simplify the subsequent exposition we now set the constant terms to zero:

$$
\begin{aligned}
G D P_{t} & =\rho G D P_{t-1}+\epsilon_{G t} \\
{\left[\begin{array}{c}
G D P_{E t} \\
G D P_{I t}
\end{array}\right] } & =\left[\begin{array}{l}
1 \\
1
\end{array}\right] G D P_{t}+\left[\begin{array}{c}
\epsilon_{E t} \\
\epsilon_{I t}
\end{array}\right]
\end{aligned}
$$

and the joint distribution of the errors is

$$
\epsilon_{t}=\left[\begin{array}{c}
\epsilon_{G t} \\
\epsilon_{E t} \\
\epsilon_{I t}
\end{array}\right] \sim \operatorname{iidN}(0, \Sigma), \quad \text { where } \quad \Sigma=\left[\begin{array}{ccc}
\Sigma_{G G} & \cdot & \cdot \\
\Sigma_{E G} & \Sigma_{E E} & \cdot \\
\Sigma_{I G} & \Sigma_{I E} & \Sigma_{I I}
\end{array}\right]
$$

Using the notation in Komunjer and Ng (2011), we write the system as

$$
\begin{aligned}
& s_{t+1}=A(\theta) s_{t}+B(\theta) \epsilon_{t+1} \\
& y_{t+1}=C(\theta) s_{t}+D(\theta) \epsilon_{t+1}
\end{aligned}
$$

where

$$
\begin{aligned}
s_{t} & =G D P_{t}, \quad y_{t}=\left[\begin{array}{c}
G D P_{E t} \\
G D P_{I t}
\end{array}\right] \\
A(\theta) & =\rho, \quad B(\theta)=\left[\begin{array}{lll}
1 & 0 & 0
\end{array}\right] \\
C(\theta) & =\left[\begin{array}{l}
\rho \\
\rho
\end{array}\right], \quad D(\theta)=\left[\begin{array}{lll}
1 & 1 & 0 \\
1 & 0 & 1
\end{array}\right]
\end{aligned}
$$

and $\theta=\left[\rho, \operatorname{vech}(\Sigma)^{\prime}\right]^{\prime}$. Note that only $A(\theta)$ and $C(\theta)$ are non-trivial functions of $\theta$. 
Assumption 1 The parameter vector $\theta$ satisfies the following conditions: (i) $\Sigma$ is positive definite; (ii) $0 \leq \rho<1$.

Because the rows of $D$ are linearly independent, Assumption 1(i) implies that $D \Sigma D^{\prime}$ is non-singular. In turn, we deduce that Assumptions 1, 2, and 4-NS of Komunjer and Ng (2011) are satisfied.

We now express the state-space system in terms of its innovation representation

$$
\begin{aligned}
s_{t+1 \mid t+1} & =A(\theta) s_{t \mid t}+K(\theta) a_{t+1} \\
y_{t+1} & =C(\theta) \hat{s}_{t \mid t}+a_{t+1},
\end{aligned}
$$

where $a_{t+1}$ is the one-step-ahead forecast error of the system whose variance we denote by $\Sigma_{a}(\theta)$. The innovation representation is obtained from the Kalman filter as follows. Suppose that conditional on time $t$ information $Y_{1: t}$ the distribution of $s_{t} \mid Y_{1: t} \sim N\left(s_{t \mid t}, P_{t \mid t}\right)$. Then the joint distribution of $\left[s_{t+1}, y_{t+1}^{\prime}\right]^{\prime}$ is

$$
\left[\begin{array}{c}
s_{t+1} \\
y_{t+1}
\end{array}\right] \mid Y_{1: T} \sim\left(\left[\begin{array}{c}
A s_{t \mid t} \\
C s_{t \mid t}
\end{array}\right],\left[\begin{array}{cc}
A P_{t \mid t} A^{\prime}+B \Sigma B^{\prime} & A P_{t \mid t} C^{\prime}+B \Sigma D^{\prime} \\
C P_{t \mid t} A^{\prime}+D \Sigma B^{\prime} & C P_{t \mid t} C^{\prime}+D \Sigma D^{\prime}
\end{array}\right]\right)
$$

In turn, the conditional distribution of $s_{t+1} \mid Y_{1: t+1}$ is

$$
s_{t+1} \mid Y_{1: t+1} \sim N\left(s_{t+1 \mid t+1}, P_{t+1 \mid t+1}\right)
$$

where

$$
\begin{aligned}
s_{t+1 \mid t+1} & =A s_{t \mid t}+\left(A P_{t \mid t} C+B \Sigma D^{\prime}\right)\left(C P_{t \mid t} C^{\prime}+D \Sigma D^{\prime}\right)^{-1}\left(y_{t}-C s_{t \mid t}\right) \\
P_{t+1 \mid t+1} & =A P_{t \mid t} A^{\prime}+B \Sigma B^{\prime}-\left(A P_{t \mid t} C^{\prime}+B \Sigma D^{\prime}\right)\left(C P_{t \mid t} C^{\prime}+D \Sigma D^{\prime}\right)^{-1}\left(C P_{t \mid t} A^{\prime}+D \Sigma B^{\prime}\right) .
\end{aligned}
$$

Now let $P$ be the matrix that solves the Riccati equation,

$$
P=A P A^{\prime}+B \Sigma B^{\prime}-\left(A P C^{\prime}+B \Sigma D^{\prime}\right)\left(C P C^{\prime}+D \Sigma D^{\prime}\right)^{-1}\left(C P A^{\prime}+D \Sigma B^{\prime}\right)
$$

and let $K$ be the Kalman gain matrix

$$
K=\left(A P C^{\prime}+B \Sigma D^{\prime}\right)\left(C P C^{\prime}+D \Sigma D^{\prime}\right)^{-1} .
$$


Then the one-step-ahead forecast error matrix is given by

$$
\Sigma_{a}=C P C^{\prime}+D \Sigma D^{\prime}
$$

Equations (A.7) to (A.9) determine the matrices that appear in the innovation-representation of the state-space system (A.6).

In order to be able to apply Proposition 1-NS of Komunjer and Ng (2011) we need to express $P, K$, and $\Sigma_{a}$ in terms of $\theta$. While solving Riccati equations analytically is in general not feasible, our system is scalar, which simplifies the calculation considerably. Replacing $A$ by $\rho$ and $P$ by $p$ such that scalars appear in lower case, and defining

$$
\Sigma_{B B}=B \Sigma B^{\prime}, \quad \Sigma_{B D}=B \Sigma D^{\prime}, \quad \text { and } \quad \Sigma_{D D}=D \Sigma D^{\prime},
$$

we can write (A.7) as

$$
p=p \rho^{2}+\Sigma_{B B}-\left(p \rho C^{\prime}+\Sigma_{B D}\right)\left(p C C^{\prime}+\Sigma_{D D}\right)^{-1}\left(p \rho C+\Sigma_{D B}\right) .
$$

Likewise,

$$
K=\left(p \rho C^{\prime}+\Sigma_{B D}\right)\left(p C C^{\prime}+\Sigma_{D D}\right)^{-1} \quad \text { and } \quad \Sigma_{a}=p C C^{\prime}+\Sigma_{D D}
$$

Because $\Sigma_{B B}-\Sigma_{B D} \Sigma_{D D}^{\prime} \Sigma_{D B}>0$ we can deduce that $p>0$. Moreover, because $A=\rho \geq 0$ and $C \geq 0$, we deduce that $K \neq 0$ and therefore Assumption 5 -NS of Komunjer and $\mathrm{Ng}$ (2011) is satisfied. According to Proposition 1-NS in Komunjer and Ng (2011), two vectors $\theta$ and $\theta_{1}$ are observationally equivalent if and only if there exists a scalar $\gamma \neq 0$ such that

$$
\begin{aligned}
A\left(\theta_{1}\right) & =\gamma A(\theta) \gamma^{-1} \\
K\left(\theta_{1}\right) & =\gamma K(\theta) \\
C\left(\theta_{1}\right) & =C(\theta) \gamma^{-1} \\
\Sigma_{a}\left(\theta_{1}\right) & =\Sigma_{a}(\theta) .
\end{aligned}
$$

Define $\theta=\left[\rho, \operatorname{vech}(\Sigma)^{\prime}\right]^{\prime}$ and $\theta_{1}=\left[\rho_{1}, \operatorname{vech}\left(\Sigma_{1}\right)^{\prime}\right]^{\prime}$. Using the definition of the scalar $A(\theta)$ in (A.5) we deduce from (A.12) that $\rho_{1}=\rho$. Since $C(\theta)$ depends on $\theta$ only through $\rho$ we can deduce from (A.14) that $\gamma=1$. Thus, given $\theta$ and $\rho$, the elements of the vector $\operatorname{vech}\left(\Sigma_{1}\right)$ 
have to satisfy conditions (A.13) and (A.15), which, using (A.11), can be rewritten as

$$
\begin{aligned}
\Sigma_{a} & =\Sigma_{a 1}=p_{1} C C^{\prime}+\Sigma_{D D 1} \\
K & =K_{1}=\left(p_{1} \rho C^{\prime}+\Sigma_{B D 1}\right) \Sigma_{a}^{-1} .
\end{aligned}
$$

Moreover, $p_{1}$ has to solve the Riccati equation (A.10):

$$
p_{1}=p_{1} \rho^{2}+\Sigma_{B B 1}-K_{0}\left(p_{1} \rho C+\Sigma_{B D}\right)
$$

Equations (A.16) to (A.18) are satisfied if and only if

$$
\begin{aligned}
p C C^{\prime}+\Sigma_{D D} & =p_{1} C C^{\prime}+\Sigma_{D D 1} \\
p \rho C^{\prime}+\Sigma_{B D} & =p_{1} \rho C^{\prime}+\Sigma_{B D 1} \\
p\left(1-\rho^{2}\right)-\Sigma_{B B} & =p_{1}\left(1-\rho^{2}\right)-\Sigma_{B B 1} .
\end{aligned}
$$

We proceed by deriving expressions for the $\Sigma_{x x}$ matrices that appear in (A.19) to (A.21):

$$
\begin{aligned}
\Sigma_{B B} & =\Sigma_{G G} \\
\Sigma_{B D} & =\left[\begin{array}{ll}
\Sigma_{G G}+\Sigma_{G E} & \Sigma_{G G}+\Sigma_{G I}
\end{array}\right] \\
\Sigma_{D D} & =\left[\begin{array}{cc}
\Sigma_{G G}+\Sigma_{E E}+2 \Sigma_{E G} & \cdot \\
\Sigma_{G G}+\Sigma_{G E}+\Sigma_{G I}+\Sigma_{E I} & \Sigma_{G G}+\Sigma_{I I}+2 \Sigma_{G I}
\end{array}\right]
\end{aligned}
$$

Without loss of generality let

$$
\Sigma_{G G 1}=\Sigma_{G G}+\left(1-\rho^{2}\right) \delta
$$

which implies that

$$
\Sigma_{B B 1}=\Sigma_{B B}+\left(1-\rho^{2}\right) \delta
$$

We now distinguish the cases $\delta=0$ and $\delta \neq 0$.

Case 1: $\delta=0$. (A.21) implies $p_{1}=p$. It follows from (A.20) that $\Sigma_{B D 1}=\Sigma_{B D}$. In turn, $\Sigma_{G E 1}=\Sigma_{G E}$ and $\Sigma_{G I 1}=\Sigma_{G I}$. Finally, to satisfy (A.19) it has to be the case that $\Sigma_{D D 1}=\Sigma_{D D}$, which implies that the remaining elements of $\Sigma$ and $\Sigma_{1}$ are identical. We conclude that $\theta_{1}=\theta$. 
Case 2: $\delta \neq 0$. (A.21) implies $p_{1}=p+\delta$. Now consider (A.20):

$$
\begin{aligned}
p \rho C^{\prime}+\Sigma_{B D}= & p \rho^{2}\left[\begin{array}{ll}
1 & 1
\end{array}\right]+\left[\begin{array}{ll}
\Sigma_{G G}+\Sigma_{G E} & \Sigma_{G G}+\Sigma_{G I}
\end{array}\right] \\
\stackrel{!}{=} & p \rho^{2}\left[\begin{array}{ll}
1 & 1
\end{array}\right]+\delta \rho^{2}\left[\begin{array}{ll}
1 & 1
\end{array}\right] \\
& +\left[\begin{array}{ll}
\Sigma_{G G}+\Sigma_{G E 1} & \Sigma_{G G}+\Sigma_{G I 1}
\end{array}\right] \\
& +\delta\left(1-\rho^{2}\right)\left[\begin{array}{ll}
1 & 1
\end{array}\right]
\end{aligned}
$$

We deduce that

$$
\Sigma_{G E 1}=\Sigma_{G E}-\delta, \quad \Sigma_{G I 1}=\Sigma_{G I}-\delta
$$

Finally, consider (A.19), which can be rewritten as

$$
0=\Sigma_{D D 1}-\Sigma_{D D}+\delta C C^{\prime}
$$

Using the previously derived expressions for $\Sigma_{D D}$ and $\Sigma_{D D 1}$ we obtain the following three conditions

$$
\begin{aligned}
& 0=\left(1-\rho^{2}\right) \delta+\left(\Sigma_{E E 1}-\Sigma_{E E}\right)-2 \delta+\rho^{2} \delta=\Sigma_{E E 1}-\Sigma_{E E}-\delta \\
& 0=\left(1-\rho^{2}\right) \delta-2 \delta+\left(\Sigma_{E I 1}-\Sigma_{E I}\right)+\rho^{2} \delta=\Sigma_{E I 1}-\Sigma_{E I}-\delta \\
& 0=\left(1-\rho^{2}\right) \delta+\left(\Sigma_{I I 1}-\Sigma_{I I}\right)-2 \delta+\rho^{2} \delta=\Sigma_{I I 1}-\Sigma_{I I}-\delta .
\end{aligned}
$$

Thus, we deduce that

$$
\Sigma_{E E 1}=\Sigma_{E E}+\delta, \quad \Sigma_{E I 1}=\Sigma_{E I}+\delta, \quad \text { and } \quad \Sigma_{I I 1}=\Sigma_{I I}+\delta
$$

Combining (A.22), (A.23), and (A.24) we find that

$$
\Sigma_{1}=\left[\begin{array}{ccc}
\Sigma_{G G}+\delta\left(1-\rho^{2}\right) & \Sigma_{G E}-\delta & \Sigma_{G I}-\delta \\
\Sigma_{G E}-\delta & \Sigma_{E E}+\delta & \Sigma_{E I}+\delta \\
\Sigma_{G I}-\delta & \Sigma_{E I}+\delta & \Sigma_{I I}+\delta
\end{array}\right]
$$

Thus, we have proved the following theorem:

Theorem A.1 Suppose Assumption 1 is satisfied. Then the two-variable model is

(i) identified if $\Sigma$ is diagonal as in section 2.1;

(ii) identified if $\Sigma$ is block-diagonal as in section 2.2; 
(iii) not identified if $\Sigma$ is unrestricted as in section 2.3;

(iv) identified if $\Sigma$ is restricted as in section 2.4.

\section{B Identification in the Three-Variable Model}

The identification analysis of the three-variable is similar to the analysis of the two-variable model in the previous section. The system is given by

$$
\begin{aligned}
G D P_{t} & =\rho G D P_{t-1}+\epsilon_{G t} \\
{\left[\begin{array}{c}
G D P_{E t} \\
G D P_{I t} \\
U_{t}
\end{array}\right] } & =\left[\begin{array}{l}
1 \\
1 \\
\lambda
\end{array}\right] G D P_{t}+\left[\begin{array}{c}
\epsilon_{E t} \\
\epsilon_{I t} \\
\epsilon_{U t}
\end{array}\right],
\end{aligned}
$$

and the joint distribution of the errors is

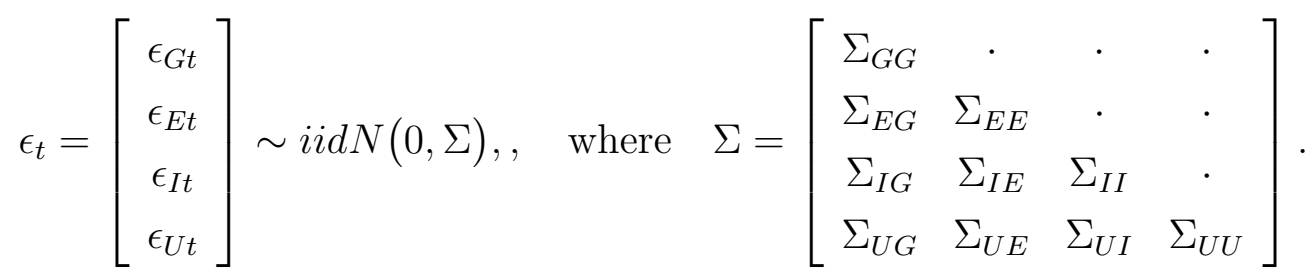

The matrices $A(\theta), B(\theta), C(\theta)$, and $D(\theta)$ are now given by

$$
\begin{aligned}
& A(\theta)=\rho, \quad B(\theta)=\left[\begin{array}{llll}
1 & 0 & 0 & 0
\end{array}\right] \\
& C(\theta)=\left[\begin{array}{c}
\rho \\
\rho \\
\lambda \rho
\end{array}\right], \quad D(\theta)=\left[\begin{array}{llll}
1 & 1 & 0 & 0 \\
1 & 0 & 1 & 0 \\
\lambda & 0 & 0 & 1
\end{array}\right],
\end{aligned}
$$

where $\theta=\left[\rho, \lambda, \operatorname{vech}(\Sigma)^{\prime}\right]^{\prime}$.

Assumption 2 The parameter vector $\theta$ satisfies the following conditions: (i) $\Sigma$ is positive definite; (ii) $0<\rho<1$; (iii) $\lambda \neq 0$; (iv) $\Sigma_{U E}=\Sigma_{U I}=0$.

Condition (A.12) implies that $\rho_{1}=\rho$. Moreover, (A.14) implies that $\gamma=1$ and that $\lambda_{1}=\lambda$ provided that $\rho \neq 0$. As for the two-variable model, we have to verify that (A.19) 
to (A.21) are satisfied. The matrices $\Sigma_{x x}$ that appear in these equations are given by

$$
\begin{aligned}
\Sigma_{B B}= & \Sigma_{G G} \\
\Sigma_{B D}= & {\left[\begin{array}{ccc}
\Sigma_{G G}+\Sigma_{G E} & \Sigma_{G G}+\Sigma_{G I} & \lambda \Sigma_{G G}+\Sigma_{G U}
\end{array}\right] } \\
\Sigma_{D D}= & {\left[\begin{array}{ccc}
\Sigma_{G G}+\Sigma_{E E}+2 \Sigma_{G E} & \cdot \\
\Sigma_{G G}+\Sigma_{G E}+\Sigma_{G I}+\Sigma_{E I} & \Sigma_{G G}+\Sigma_{I I}+2 \Sigma_{G I} & . \\
\lambda \Sigma_{G G}+\lambda \Sigma_{G E}+\Sigma_{G U} & \lambda \Sigma_{G G}+\lambda \Sigma_{G I}+\Sigma_{G U} & \lambda^{2} \Sigma_{G G}+2 \lambda \Sigma_{G U}+\Sigma_{U U}
\end{array}\right] . }
\end{aligned}
$$

Without loss of generality, let

$$
\Sigma_{G G, 1}=\Sigma_{G G}+\left(1-\rho^{2}\right) \delta
$$

which implies that

$$
\Sigma_{B B, 1}=\Sigma_{B B}+\left(1-\rho^{2}\right) \delta
$$

Case 1: $\delta=0$. (A.21) implies $p_{1}=p$. It follows from (A.20) that $\Sigma_{B D, 1}=\Sigma_{B D}$. In turn, $\Sigma_{G E, 1}=\Sigma_{G E}, \Sigma_{G I, 1}=\Sigma_{G I}$, and $\Sigma_{G U, 1}=\Sigma_{G U}$. Finally, to satisfy (A.17) it has to be the case that $\Sigma_{D D, 1}=\Sigma_{D D}$, which implies that the remaining elements of $\Sigma$ and $\Sigma_{1}$ are identical for the two parameterizations. We conclude that it has to be the case that $\theta_{1}=\theta$.

Case 2: $\delta \neq 0$. (A.21) implies $p_{1}=p+\delta$. Now consider (A.20):

$$
\begin{aligned}
& p \rho C^{\prime}+\Sigma_{B D}=p \rho^{2}\left[\begin{array}{lll}
1 & 1 & \lambda
\end{array}\right]+\left[\begin{array}{lll}
\Sigma_{G G}+\Sigma_{G E} & \Sigma_{G G}+\Sigma_{G I} & \lambda \Sigma_{G G}+\Sigma_{G U}
\end{array}\right] \\
& \stackrel{!}{=} p \rho^{2}\left[\begin{array}{lll}
1 & 1 & \lambda
\end{array}\right]+\delta \rho^{2}\left[\begin{array}{lll}
1 & 1 & \lambda
\end{array}\right] \\
& +\left[\begin{array}{lll}
\Sigma_{G G}+\Sigma_{G E, 1} & \Sigma_{G G}+\Sigma_{G I, 1} & \lambda \Sigma_{G G}+\Sigma_{G U, 1}
\end{array}\right] \\
& +\left(1-\rho^{2}\right) \delta\left[\begin{array}{lll}
1 & 1 & \lambda
\end{array}\right] \text {. }
\end{aligned}
$$

We deduce that

$$
\Sigma_{G E, 1}=\Sigma_{G E}-\delta, \quad \Sigma_{G I, 1}=\Sigma_{G I}-\delta, \quad \Sigma_{G U, 1}=\Sigma_{G U}-\delta
$$

Finally, consider (A.19), which can be rewritten as

$$
0=\Sigma_{D D, 1}-\Sigma_{D D}+\delta C C^{\prime}
$$


Using the previously derived expressions for $\Sigma_{D D}$ and $\Sigma_{D D 1}$ we obtain the following five conditions

$$
\begin{aligned}
0 & =\left(1-\rho^{2}\right) \delta+\left(\Sigma_{E E 1}-\Sigma_{E E}\right)-2 \delta+\rho^{2} \delta=\Sigma_{E E 1}-\Sigma_{E E}-\delta \\
0 & =\left(1-\rho^{2}\right) \delta-2 \delta+\left(\Sigma_{E I 1}-\Sigma_{E I}\right)+\rho^{2} \delta=\Sigma_{E I 1}-\Sigma_{E I}-\delta \\
0 & =\left(1-\rho^{2}\right) \delta+\left(\Sigma_{I I 1}-\Sigma_{I I}\right)-2 \delta+\rho^{2} \delta=\Sigma_{I I 1}-\Sigma_{I I}-\delta \\
0 & =\lambda\left(1-\rho^{2}\right) \delta-\lambda \delta-\delta+\lambda \rho^{2} \delta=\delta \\
0 & =\lambda^{2}\left(1-\rho^{2}\right) \delta-2 \lambda \delta+\left(\Sigma_{U U 1}-\Sigma_{U U}\right)+\lambda^{2} \rho^{2} \delta=\Sigma_{U U 1}-\Sigma_{U U}-\lambda(2-\lambda) \delta .
\end{aligned}
$$

Thus, we deduce that

$$
\delta=0, \quad, \Sigma_{E E 1}=\Sigma_{E E}, \quad \Sigma_{E I 1}=\Sigma_{E I}, \quad \Sigma_{I I 1}=\Sigma_{I I}, \quad \text { and } \quad \Sigma_{U U 1}=\Sigma_{U U} .
$$

This proves the following theorem:

Theorem B.1 Suppose Assumption 2 is satisfied. Then the three-variable model is identified. 


\section{References}

Aruoba, B. (2008), "Data Revisions are not Well-Behaved," Journal of Money, Credit and Banking, 40, 319-340.

Aruoba, S.B., F.X. Diebold, J. Nalewaik, F Schorfheide, and D. Song (2012), "Improving GDP Measurement: A Forecast Combination Perspective," In X. Chen and N. Swanson (eds.), Recent Advances and Future Directions in Causality, Prediction, and Specification Analysis: Essays in Honour of Halbert L. White Jr., Springer, 1-26.

Carter, C.K. and R. Kohn (1994), "On Gibbs Sampling for State Space Models," Biometrika, $81,541-553$.

Diebold, F.X. and L. Kilian (2001), "Measuring Predictability: Theory and Macroeconomic Applications," Journal of Applied Econometrics, 16, 657-669.

Durbin, J. and S.J. Koopman (2001), Time Series Analysis by State Space Methods, Oxford: Oxford University Press.

Edwards, C.L. and E.P. Howrey (1991), "A 'True' Time Series and Its Indicators: An Alternative Approach," Journal of the American Statistical Association, 86, 878-882.

Faust, J., J.H. Rogers, and J.H. Wright (2005), "News and Noise in G-7 GDP Announcements," Journal of Money, Credit and Banking, 37, 403-417.

Fixler, D.J. and J.J. Nalewaik (2009), "News, Noise, and Estimates of the "True" Unobserved State of the Economy," Manuscript, Bureau of Labor Statistics and Federal Reserve Board.

Fleischman, C.A. and J.M. Roberts (2011), "A Multivariate Estimate of Trends and Cycles," Manuscript, Federal Reserve Board.

Gartaganis, A.J. and A.S. Goldberger (1955), "A Note on the Statistical Discrepancy in the National Accounts," Econometrica, 23, 166-173.

Geweke, J.F. (1977), "The Dynamic Factor Analysis of Economic Time Series Models," In D. Aigner and A. Goldberger (eds.), Latent Variables in Socioeconomic Models, North Holland, 365-383.

Harding, D. and R. Scutella (1996), "Efficient Estimates of GDP," Unpublished Seminar Notes, La Trobe University, Australia. 
Jacobs, J.P.A.M. and S. van Norden (2011), "Modeling Data Revisions: Measurement Error and Dynamics of "True" Values," Journal of Econometrics, 161, 101-109.

Kishor, N.K. and E.F. Koenig (2011), "VAR Estimation and Forecasting When Data Are Subject to Revision," Journal of Business and Economic Statistics, in press.

Komunjer, I. and S. Ng (2011), "Dynamic Identification of Dynamic Stochastic General Equilibrium Models," Econometrica, 79, 1995-2032.

Mankiw, N.G., D.E. Runkle, and M.D. Shapiro (1984), "Are Preliminary Announcements of the Money Stock Rational Forecasts?" Journal of Monetary Economics, 14, 15-27.

Mankiw, N.G. and M.D. Shapiro (1986), "News or Noise: An Analysis of GNP Revisions," Survey of Current Business, May, 20-25.

Nalewaik, J. (2012), "Estimating Probabilities of Recession in Real Time with GDP and GDI," Journal of Money, Credit and Banking, 44, 235-253.

Nalewaik, J. and E. Pinto (2012), "The Response of Capital Goods Shipments to Demand over the Business Cycle," Manuscript, Federal Reserve Board.

Nalewaik, J.J. (2010), "The Income- and Expenditure-Side Estimates of U.S. Output Growth," Brookings Papers on Economic Activity, 1, 71-127 (with discussion).

Rapach, D.E., J.K. Strauss, and G. Zhou (2010), "Out-of-Sample Equity Premium Prediction: Combination Forecasts and Links to the Real Economy," Review of Financial Studies, 23, 821-862.

Sargent, T.J. and C.A. Sims (1977), "Business Cycle Modeling Without Pretending to Have too Much a Priori Theory," In C.A. Sims (ed.), New Methods in Business Cycle Research: Proceedings from a Conference, Federal Reserve Bank of Minneapolis, 45-109.

Smith, R.J., M.R. Weale, and S.E. Satchell (1998), "Measurement Error with Accounting Constraints: Point and Interval Estimation for Latent Data with an Application to U.K. Gross Domestic Product," The Review of Economic Studies, 65, 109-134.

Stone, R., D.G. Champernowne, and J.E. Meade (1942), "The Precision of National Income Estimates," Review of Economic Studies, 9, 111-125. 
Watson, M.W. and R.F. Engle (1983), "Alternative Algorithms for the Estimation of Dynamic Factor, MIMIC and Varying Coefficient Regression Models," Journal of Econometrics, 23, 385-400. 